\title{
Degrees of Guaranteed Envy-Freeness in Finite Bounded Cake-Cutting Protocols*
}

\author{
Claudia Lindner and Jörg Rothe \\ Institut für Informatik \\ Heinrich-Heine-Universität Düsseldorf \\ 40225 Düsseldorf, Germany
}

October 19, 2009

\begin{abstract}
Cake-cutting protocols aim at dividing a "cake" (i.e., a divisible resource) and assigning the resulting portions to several players in a way that each of the players feels to have received a "fair" amount of the cake. An important notion of fairness is envy-freeness: No player wishes to switch the portion of the cake received with another player's portion. Despite intense efforts in the past, it is still an open question whether there is a finite bounded envy-free cake-cutting protocol for an arbitrary number of players, and even for four players.

We introduce the notion of degree of guaranteed envy-freeness (DGEF) as a measure of how good a cake-cutting protocol can approximate the ideal of envy-freeness while keeping the protocol finite bounded (trading being disregarded). We propose a new finite bounded proportional protocol for any number $n \geq 3$ of players, and show that this protocol has a DGEF of $1+\left[n^{2} / 2\right]$. This is the currently best DGEF among known finite bounded cake-cutting protocols for an arbitrary number of players. We will make the case that improving the DGEF even further is a tough challenge, and determine, for comparison, the DGEF of selected known finite bounded cake-cutting protocols.
\end{abstract}

\section{Introduction}

Fair allocation of goods or resources among various agents is a central task in multiagent systems and other fields. The specific setting where just one divisible resource is to be divided fairly is commonly referred to as cake-cutting, and agents are called players in this setting. Research in the area of cake-cutting started off in the 1940s with the pioneering work of Steinhaus [Ste48] who, to the best of our knowledge, was the first to introduce the problem of fair division. Dividing a

\footnotetext{
*Supported in part by the DFG under grants RO 1202/12-1 (within the European Science Foundation's EUROCORES program LogICCC: "Computational Foundations of Social Choice") and RO 1202/11-1 and by the Alexander von Humboldt Foundation's TransCoop program. A preliminary version of this paper is to appear in the proceedings of the 5th Workshop on Internet \& Network Economics (WINE-2009), December 2009. Work done in part while the second author was visiting the University of Rochester.
} 
good (or a resource) fairly among several players such that each of them is satisfied with the portion received is of central importance in many fields. In the last 60 years this research area has developed vividly, spreading out into various directions and with applications in areas as diverse as economics, mathematics, computer science, and psychology. While some lines of this research seek to find reasonable interpretations of what "fairness" really stands for and how to measure it [Fol67, FK74, Cha86], others are concerned more with proofs of existence or impossibility theorems regarding fair division [Var74, Wel85, Aki95], or with the development of new cake-cutting procedures [BT95, Str80, RW98, BB04] and, relatedly, with the analysis of their complexity relative to both upper and lower bounds [MIBK03, WS07, Pro09]. Since cake-cutting procedures involve several parties (namely, the players), they are also referred to as "protocols."

Cake-cutting protocols aim at achieving a fair division of an infinitely divisible resource among $n$ players, who each may have different preferences for (and thus different valuations of) different parts of the resource. In this paper, we focus on (a notion of) fairness in finite bounded cakecutting protocols. Many cake-cutting protocols are known today, both finite and continuous ones. While a finite protocol always provides a solution after only a finite number of decisions have been made, a continuous protocol could potentially run forever. Among finite protocols, one can further distinguish between bounded and unbounded ones. A finite bounded cake-cutting protocol is present if we know in advance that a certain number of steps (that may depend on the number of players) will suffice to divide the resource fairly-independently of how the players may value distinct parts of the resource in a particular case and independently of the strategies chosen by the players. In contrast, in finite unbounded cake-cutting protocols, we cannot predict an upper bound on how many steps will be required to achieve the same goal. Aiming to apply cake-cutting procedures to real-world scenarios, it is important to develop fair finite bounded cake-cutting protocols. In this context, "fairness" is often interpreted as meaning "envy-freeness." A division is envy-free if no player has an incentive to switch his or her portion with the portion any other player received.

Steinhaus [Ste49] proved that for any number of players an envy-free division of a single divisible good always exists. However, the current state of the art-after six decades of intense research-is that for arbitrary $n$, and even for $n=4$, the development of finite bounded envy-free cake-cutting protocols still appears to be out of reach, and a big challenge for future research. For $n>3$ players, hardly any envy-free cake-cutting protocol is known, and the ones that are known are either finite unbounded or continuous (see, e.g., [BT95, RW97, BTZ97]). Though, from an implementation perspective, finite bounded protocols are the ones that are most desirable. Recently, Stromquist [Str07] has shown that for more than two players there is no finite cake-cutting protocol that provides an envy-free division when all portions are required to consist of contiguous pieces.

Our goal in this paper is to look for compromises that can be made with respect to envy-freeness while keeping the protocol finite bounded: We propose an approach to evaluate finite bounded (yet possibly non-envy-free) cake-cutting protocols with respect to their "degree of guaranteed envyfreeness" (DGEF). Informally put, this notion provides a measure of how good such a protocol can approximate the (possibly for this particular protocol unreachable) ideal of envy-freeness in terms of the number of envy-free-relations that are guaranteed to exist even in the worst case.

This paper is organized as follows. After defining some basic notions in Section 2, we introduce the notion of degree of guaranteed envy-freeness, and specify the DGEF for some well-known finite bounded proportional cake-cutting protocols in Section 3. In Section 4 we present a new 
finite bounded proportional cake-cutting protocol with an enhanced degree of guaranteed envyfreeness, compared with the proportional protocols mentioned in Section 3. This new cake-cutting protocol makes use of parallelization in order to include as many matching valuations (in terms of not raising envy) as possible. Section 5 briefly describes those protocols mentioned in Section 3 , and determines their degree of guaranteed envy-freeness via a detailed analysis. In Section 6 , we compare the DGEF approach with related work, and show that even small steps toward the development of cake-cutting protocols with an enhanced DGEF are of significance. Finally, we conclude in Section 7 that our approach extends the scope for the development of new finite bounded cake-cutting protocols by "approximating" envy-freeness instead of insisting on it.

\section{Preliminaries and Notation}

Cake-cutting is about dividing a cake into portions that are assigned to the players such that each of them feels, according to his or her individual valuation of the portions, to have received a fair amount of the cake 1 The cake is assumed to be infinitely divisible, and can be divided into arbitrary pieces without losing any of its value. Moreover, we assume the cake to be heterogeneous 2 This assumption can be made without loss of generality, as any cake-cutting protocol providing a "fair" division of a heterogeneous resource can be applied in the same way to a homogeneous one [BT96]. Given $n$ players, cake $C$ is to be divided into $n$ portions that are to be distributed among the players so as to satisfy each of them. A portion is not necessarily a single piece of cake but rather can be a collection of disjoint, possibly noncontiguous pieces of $C$. Furthermore, all players may have different individual valuations of the single pieces of the cake. For example, one player may prefer the pieces with the chocolate topping, whereas another player may prefer the pieces with the cherry topping.

More formally, cake $C$ is represented by the unit interval $[0,1]$ of real numbers. By performing cuts, $C$ is divided into $m$ pieces $c_{k}, 1 \leq k \leq m$ : Each player $p_{i}, 1 \leq i \leq n$, assigns value $v_{i}\left(c_{k}\right)=$ $v_{i}\left(x_{k}, y_{k}\right)$ to piece $c_{k} \subseteq C$, where $c_{k}$ is represented by the subinterval $\left[x_{k}, y_{k}\right] \subseteq[0,1]$ and $p_{i}$ 's valuation function $v_{i}$ maps subintervals of $[0,1]$ to real numbers in $[0,1]$. We require each valuation function $v_{i}$ to satisfy the following properties:

1. Normalization: $v_{i}(0,1)=1$.

2. Positivity: $\exists^{3}$ For all $c_{k} \subseteq C, c_{k} \neq \emptyset$, we have $v_{i}\left(c_{k}\right)>0$.

3. Additivity: For all $c_{k}, c_{\ell} \subseteq C, c_{k} \cap c_{\ell}=\emptyset$, we have $v_{i}\left(c_{k}\right)+v_{i}\left(c_{\ell}\right)=v_{i}\left(c_{k} \cup c_{\ell}\right)$.

\footnotetext{
${ }^{1}$ As is common, "cake" will be used as a metaphor for the resource or the good to be divided.

${ }^{2}$ Consider, for example, a cake with cherry, chocolate, and strawberry toppings. A player may value, say, the pieces with strawberry toppings higher than those with cherry toppings.

${ }^{3}$ The literature is a bit ambiguous regarding this assumption. Some papers require the players' values for nonempty pieces of cake to be nonnegative (i.e., $v_{i}\left(c_{k}\right) \geq 0$ ) instead of positive. For example, Robertson and Webb [RW98] and Woeginger and Sgall [WS07] require nonnegative values for nonempty pieces of cake, whereas positive values for such pieces are required by Brams and Taylor [BT96], Brams, Jones, and Klamler [BJK07], and Weller [Wel85].
} 
4. Divisibility. 4 For all $c_{k} \subseteq C$ and for each $\alpha, 0 \leq \alpha \leq 1$, there exists some $c_{\ell} \subseteq c_{k}$ such that $v_{i}\left(c_{\ell}\right)=\alpha \cdot v_{i}\left(c_{k}\right)$.

Note that, to simplify notation, we write $v_{i}\left(x_{k}, y_{k}\right)$ instead of $v_{i}\left(\left[x_{k}, y_{k}\right]\right)$ for intervals $\left[x_{k}, y_{k}\right] \subseteq[0,1]$. Due to Footnote 4 no ambiguity can arise. For each $[x, y] \subseteq[0,1]$, define $\|[x, y]\|=y-x$. For any real number $x,\lfloor x\rfloor$ denotes the greatest integer not exceeding $x$, and $\lceil x\rceil$ denotes the least integer not smaller than $x$.

The assumption that $C$ is heterogeneous formally means that subintervals of $[0,1]$ having equal size can be valued differently by the same player. Moreover, distinct players may value one and the same piece of the cake differently, i.e., their individual valuation functions will in general be distinct. Every player knows only the value of (arbitrary) pieces of $C$ corresponding to his or her own valuation function. Players do not have any knowledge about the valuation functions of other players.

A division of $C$ is an assignment of disjoint and nonempty portions $C_{i} \subseteq C$, where $C=\bigcup_{i=1}^{n} C_{i}=$ $\bigcup_{k=1}^{m} c_{k}$, to the players such that each player $p_{i}$ receives a portion $C_{i} \subseteq C$ consisting of at least one nonempty piece $c_{k} \subseteq C$. The goal of a cake-cutting division is to assign the portions to the players in as fair a way as possible. There are different interpretations, though, of what "fair" might mean. To distinguish between different degrees of fairness, the following notions have been introduced in the literature (see, e.g., Robertson and Webb [RW98]):

Definition 2.1 Let $v_{1}, v_{2}, \ldots, v_{n}$ be the valuation functions of the $n$ players. A division of cake $C=\bigcup_{i=1}^{n} C_{i}$, where $C_{i}$ is the ith player's portion, is said to be:

1. simple fair (a.k.a. proportional) if and only if for each $i, 1 \leq i \leq n$, we have $v_{i}\left(C_{i}\right) \geq 1 / n$;

2. strong fair if and only if for each $i, 1 \leq i \leq n$, we have $v_{i}\left(C_{i}\right)>1 / n$;

3. envy-free if and only if for each $i$ and $j, 1 \leq i, j \leq n$, we have $v_{i}\left(C_{i}\right) \geq v_{i}\left(C_{j}\right)$.

A cake-cutting protocol describes an interactive procedure for obtaining a division of a given cake, without having any information on the valuation functions of the players involved. Each protocol is characterized by a set of rules and a set of strategies (see, e.g., Brams and Taylor [BT96]). The rules just determine the course of action, such as a request to cut the cake, whereas the strategies define how to achieve a certain degree of fairness, e.g., by advising the players where to cut the cake. If all players obey the protocol, it is guaranteed that every player receives a "fair" portion of the cake. Cake-cutting protocols are characterized according to the degree of fairness of the divisions obtained:5

Definition 2.2 A cake-cutting protocol is said to be simple fair (or proportional), strong fair, and envy-free, respectively, if every division obtained (i.e., regardless of which valuation functions the players have) is simple fair (or proportional), strong fair, and envy-free, respectively, provided that all players follow the rules and strategies of the protocol.

\footnotetext{
${ }^{4}$ Divisibility implies that for each $x \in[0,1], v_{i}(x, x)=0$. That is, isolated points are valued 0 , and open intervals have the same value as the corresponding closed intervals.

${ }^{5}$ In addition to the fairness criteria given here, other fairness criteria may be reasonable as well and have been proposed in the literature (see, e.g., Robertson and Webb [RW98]).
} 
Apparently, every division obtained by either a strong fair cake-cutting protocol or by an envyfree cake-cutting protocol is simple fair as well (i.e., every strong fair or envy-free cake-cutting protocol can be classified as being simple fair, too). Moreover, every simple fair cake-cutting protocol can easily be applied to the case when there are unequal shares to be assigned, though with respect to rational ratios only. Informally speaking, this can be done by cloning players and their valuation functions so as to have in total as many players as the smallest common denominator specifies (see, e.g., [RW98]).

\section{Degrees of Guaranteed Envy-Freeness for Proportional Protocols}

As mentioned in the introduction, the design of envy-free cake-cutting protocols for any number $n$ of players seems to be quite a challenge. For $n \leq 3$ players, a number of protocols that always provide envy-free divisions have been published, both finite (bounded and unbounded) and continuous ones [Str80, BT96, RW98, BB04]. However, to the best of our knowledge, up to date no finite bounded cake-cutting protocol for $n>3$ players is known to always provide an envy-free division. For practical purposes, it would be most desirable to have finite bounded cake-cutting protocols that always provide divisions as fair as possible. In this regard, it is questionable whether the advantage of always having an envy-free rather than just a proportional division would be big enough to justify the lack of finite boundedness. It may be worthwhile to be content with a certain lower degree of envy-freeness, rather than insisting on complete envy-freeness, for the benefit of having a finite bounded protocol in exchange.

In this paper, we propose an approach that weakens the concept of envy-freeness for the purpose of keeping protocols finite bounded. On the one hand, in Section 3.2 we are concerned with known simple fair (i.e., proportional) cake-cutting protocols that are finite bounded, and determine their degree of guaranteed envy-freeness, a notion to be introduced in Section 3.1 (see Definition 3.2). On the other hand, in Section 4 we propose a new finite bounded proportional cake-cutting protocol that - compared with the known protocols- has an enhanced degree of guaranteed envy-freeness.

\subsection{Degrees of Guaranteed Envy-Freeness}

When investigating the degree of envy-freeness of a cake-cutting protocol for $n$ players, for each player $p_{i}, 1 \leq i \leq n$, the value of his or her portion needs to be compared to the values of the $n-1$ other portions (according to the measure of player $\left.p_{i}\right) 6$ Thus, $n(n-1)$ pairwise relations need to be investigated in order to determine the degree of envy-freeness of a cake-cutting protocol for $n$ players. A player $p_{i}$ envies another player $p_{j}, 1 \leq i, j \leq n, i \neq j$, when $p_{i}$ prefers player $p_{j}$ 's portion to his or her own. If $p_{i}$ envies $p_{j}$, we call the relation from $p_{i}$ to $p_{j}$ an envy-relation; otherwise, we call it an envy-free-relation.

Definition 3.1 Consider a division of cake $C=\bigcup_{i=1}^{n} C_{i}$ for a set $P=\left\{p_{1}, p_{2}, \ldots, p_{n}\right\}$ of players, where $v_{i}$ is $p_{i}$ 's valuation function and $C_{i}$ is $p_{i}$ 's portion.

1. An envy-relation for this division (denoted by $\Vdash$ ) is a binary relation on $P$. Player $p_{i}$ envies

\footnotetext{
${ }^{6}$ We will use "valuation" and "measure" interchangeably.
} 
player $p_{j}, 1 \leq i, j \leq n, i \neq j$, if and only if $v_{i}\left(C_{i}\right)<v_{i}\left(C_{j}\right)$. We write $p_{i} \Vdash p_{j}$.

2. An envy-free-relation for this division (denoted by $\nVdash$ ) is a binary relation on $P$. Player $p_{i}$ does not envy player $p_{j}, 1 \leq i, j \leq n, i \neq j$, if and only if $v_{i}\left(C_{i}\right) \geq v_{i}\left(C_{j}\right)$. We write $p_{i} \nVdash p_{j}$.

The following properties of envy-relations and envy-free-relations are worth mentioning 77 No player can envy him- or herself, i.e., envy-relations are irreflexive: The inequality $v_{i}\left(C_{i}\right)<v_{i}\left(C_{i}\right)$ never holds. Thus, we trivially have that $v_{i}\left(C_{i}\right) \geq v_{i}\left(C_{i}\right)$ always holds. However, when counting envy-free-relations for a given division, we will disregard these trivial envy-free-relations $p_{i} \nVdash p_{i}$, $1 \leq i \leq n$, throughout the paper.

Furthermore, neither envy-relations nor envy-free-relations need to be transitive. This is due to the fact that each player values every piece of the cake according to his or her own valuation function. The valuation functions of different players will be distinct in general. For example, given three distinct players $p_{i}, p_{j}$, and $p_{k}$ with valuations $v_{i}\left(C_{i}\right)<v_{i}\left(C_{j}\right)$ and $v_{j}\left(C_{j}\right)<v_{j}\left(C_{k}\right)$, we have that $p_{i} \Vdash p_{j}$ and $p_{j} \Vdash p_{k}$. However, these valuations do not provide any information about player $p_{i}$ 's valuation of portion $C_{k}$, so we cannot conclude that $p_{i} \Vdash p_{k}$. An analogous argument applies to envy-free-relations.

The above observations imply that envy-relations and envy-free-relations are either one-way or two-way, i.e., it is possible that:

1. two players envy each other $\left(p_{i} \Vdash p_{j}\right.$ and $\left.p_{j} \Vdash p_{i}\right)$, which we refer to as "two-way envy,"

2. neither of two players envies the other ( $p_{i} \nVdash p_{j}$ and $p_{j} \nVdash p_{i}$ ), which we refer to as "two-way envy-freeness," and

3. one player envies another player but is not envied by this other player $\left(p_{i} \Vdash p_{j}\right.$ and $\left.p_{j} \nVdash p_{i}\right)$, which we refer to as both "one-way envy" (from $p_{i}$ to $p_{j}$ ) and "one-way envy-freeness" (from $p_{j}$ to $\left.p_{i}\right)$.

Assuming that all players are following the rules and strategies, some cake-cutting protocols always guarantee an envy-free division (i.e., they always find an envy-free division of the cake), whereas others do not. Only protocols that guarantee an envy-free division in every case, even in the worst case (in terms of the players' valuation functions), are considered to be envy-free. Note that an envy-free division may be obtained by coincidence, just because the players have matching valuation functions that avoid envy, and not because envy-freeness is enforced by the rules and strategies of the cake-cutting protocol used. In the worst case, however, when the players have totally nonconforming valuation functions, an envy-free division would not just happen by

\footnotetext{
${ }^{7}$ Various analogs of envy-relations and envy-free-relations have also been studied, from an economic perspective, in the different context of multiagent allocation of indivisible resources. In particular, Feldman and Weiman [FW79] consider "non-envy relations" (which are similar to our notion of envy-free-relations) and mention that these are not necessarily transitive. Chauduri [Cha85] introduces "envy-relations" and mentions that these are irreflexive and not necessarily transitive. Despite some similarities, their notions differ from ours, both in their properties and in the way properties holding for their and our notions are proven. For example, Chauduri [Cha85] notes that mutual envy cannot occur in a market equilibrium, i.e., in this case his "envy-relations" are asymmetric, which is in sharp contrast to two-way envy being allowed for our notion.
} 
coincidence, but needs to be enforced by the rules and strategies of the protocol. An envy-freerelation is said to be guaranteed if it exists even in the worst case.

We now define the degree of guaranteed envy-freeness in relation to the problem of cake-cutting.

Definition 3.2 For $n \geq 1$ players, the degree of guaranteed envy-freeness (DGEF, for short) of a given proportional 8 cake-cutting protocol is defined to be the maximum number of envy-freerelations that exist in every division obtained by this protocol (provided that all players follow the rules and strategies of the protocol), i.e., the DGEF (which is expressed as a function of $n$ ) is the number of envy-free-relations that can be guaranteed even in the worst case.

By a slight abuse of notation, we will sometimes speak of the number of guaranteed envyfree-relations (rather than of the guaranteed number of envy-free-relations). When we do so, let us remind the reader that what matters is the total number of envy-free-relations that exist in the worst case, and not the identification of specific envy-free-relations. Moreover, for technical reasons (see the proof of Lemma 5.5), we also consider the case that there is only one player (i.e., $n=1$ ). Note, however, that in this case the DGEF of any cake-cutting protocol is trivially zero, since we disregard the trivial envy-free-relation $p_{1} \nVdash p_{1}$.

Definition 3.2 is based on the idea of weakening the notion of fairness in terms of envy-freeness in order to obtain cake-cutting protocols that are fair (though perhaps non-envy-free) and finite bounded, where the fairness level of a protocol is given by its degree of guaranteed envy-freeness. The higher the degree of guaranteed envy-freeness the fairer the protocol.

\subsection{Degrees of Guaranteed Envy-Freeness in Proportional Cake-Cutting Protocols}

We now give an upper and a lower bound on the degree of guaranteed envy-freeness for proportional cake-cutting protocols. For comparison, note that Feldman and Kirman [FK74] observed that, for any division, the number of envy-relations is always between zero and $n(n-1)$; zero if everyone is happy with his or her share of the cake, and $n(n-1)$ if everyone is envious of everyone else.

Proposition 3.3 Let $d(n)$ be the degree of guaranteed envy-freeness of a proportional cake-cutting protocol for $n \geq 2$ players. It holds that $n \leq d(n) \leq n(n-1)$.

Proof. If $n=2$ then we obviously have $d(2)=2$, and this will be the case exactly if both players value their share of the cake as being at least $1 / 2$. Note that this case, $n=2=d(n)$, reflects the fact that every proportional protocol for two players is envy-free.

So, we now assume that $n \geq 3$. As stated above, we disregard the trivial envy-free-relation $p_{i} \nVdash p_{i}$ for each $i, 1 \leq i \leq n$. Consequently, each player can have at most $n-1$ envy-free-relations, one to each of the other players, which gives a total of at most $n(n-1)$ guaranteed envy-freerelations. This proves the upper bound: $d(n) \leq n(n-1)$.

To prove the lower bound, note that, by definition, in a proportional division every player $p_{i}, 1 \leq$ $i \leq n$, regards his or her portion being of value at least $1 / n$, i.e., $v_{i}\left(C_{i}\right) \geq 1 / n$. Thus, $v_{i}\left(C-C_{i}\right) \leq(n-1) / n$

\footnotetext{
${ }^{8}$ The DGEF should be restricted to proportional protocols only, since otherwise the DGEF may overstate the actual level of fairness, e.g., if all the cake is given to a single player.
} 
for each $i$. We will now prove that this implies that none of the players can envy each of the $n-1$ other players at the same time. We show this for player $p_{1}$; the argument is analogous for the other players. So, assume that $p_{1}$ envies some other player, say $p_{2}$. Thus $v_{1}\left(C_{2}\right)>v_{1}\left(C_{1}\right) \geq 1 / n$. It follows that $p_{1}$ values the remaining cake $\left(C-C_{1}\right)-C_{2}$ as being less than $(n-2) / n$, i.e., $v_{1}\left(\left(C-C_{1}\right)-C_{2}\right)<$ $(n-2) / n$. Consequently, there is no way to divide the remaining cake $\left(C-C_{1}\right)-C_{2}$ into $n-2$ portions $C_{3}, C_{4}, \ldots, C_{n}$ such that for each $i, 3 \leq i \leq n$, we have $v_{1}\left(C_{i}\right) \geq 1 / n$. Hence, there must be at least one player $p_{j}, 3 \leq j \leq n$, such that $v_{1}\left(C_{j}\right)<1 / n \leq v_{1}\left(C_{1}\right)$, so $p_{1} \nVdash p_{j}$. Considering all $n$ players, this gives at least $n$ guaranteed envy-free-relations for a proportional protocol, so $d(n) \geq n$.

The degree of fairness of a division obtained by applying a proportional cake-cutting protocol highly depends on the rules of this protocol. Specifying and committing to appropriate rules often increases the degree of guaranteed envy-freeness, whereas the lack of such rules jeopardizes it in the sense that the number of guaranteed envy-free-relations may be limited to the worst-case minimum of $n$ as stated in Proposition 3.3. In this context, "appropriate rules" are those that involve the players' evaluations of other players' portions and of pieces that still are to be assigned. Concerning a particular piece of cake, involving the evaluations of as many players as possible in the allocation process helps to keep the number of envy-relations to be created low, since this allows to determine early on whether a planned allocation may later turn out to be disadvantageous - and thus allows to take adequate countermeasures. In contrast, omitting mutual evaluations means to forego additional knowledge that could turn out to be most valuable later on.

For example, say player $p_{i}$ is going to get assigned piece $c_{j}$. If the protocol asks all other players to evaluate piece $c_{j}$ according to their measures, all envy-relations to be created by the assignment of piece $c_{j}$ to player $p_{i}$ can be identified before the actual assignment and thus countermeasures (such as trimming piece $c_{j}$ ) can be undertaken. However, if the protocol requires no evaluations on behalf of the other players, such envy-relations cannot be identified early enough to prevent them from happening.

Lemma 3.4 Let a proportional cake-cutting protocol for $n \geq 2$ players be given. If the rules of the protocol require none of the players to value any of the other players' portions, then the degree of guaranteed envy-freeness is $n$ (i.e., each player is guaranteed only one envy-free-relation).

Proof. Having a certain number of guaranteed envy-free-relations means to have at least this number in any case, even in the worst case. For $n=2$, proportionality implies envy-freeness, so the worst case is the best case. For $n \geq 3$ players, consider the following scenario. Given a division of cake $C=\bigcup_{i=1}^{n} C_{i}$, without any restrictions other than aiming at a proportional division (i.e., the rules of the protocol require none of the players to value any of the other players' portions), we set the valuation functions of the players as follows. For each $i, 1 \leq i \leq n$, player $p_{i}$ values portion $C_{i}$ to be worth exactly $n / n^{2}=1 / n, p_{i}$ values exactly one portion $C_{j}, i \neq j$, to be worth exactly $2 / n^{2}<1 / n$, and $p_{i}$ values each of the $n-2$ remaining portions $C_{k},\|\{i, j, k\}\|=3$, to be worth exactly $(n+1) / n^{2}>1 / n$. These valuations make this division proportional, as every player values his or her portion to be worth exactly $1 / n$. Moreover, each player has $n-2$ envy-relations and just one guaranteed envyfree-relation, the latter of which is due to Proposition 3.3. Hence, if the rules of the protocol require none of the players to value any of the other players' portions, then no more than $n$ envy-freerelations can be guaranteed by the given proportional cake-cutting protocol in the worst case. 
The argument in the proof of Lemma 3.4 will be used to prove the upper bounds on the DGEF of the proportional cake-cutting protocols considered in Theorem 3.5 (see also Lemmas 5.1 through 5.6) and Theorems 4.2 and 4.9

An envy-free cake-cutting protocol for $n$ players guarantees that no player $p_{i}$ envies any other player $p_{j}$, i.e., $v_{i}\left(C_{i}\right) \geq v_{i}\left(C_{j}\right)$ for all $i, j$ with $1 \leq i, j \leq n$, in each division $C=\bigcup_{i=1}^{n} C_{i}$ obtained by this protocol. This is the case exactly if the protocol meets the upper bound on the degree of guaranteed envy-freeness given in Proposition 3.3. That is, a cake-cutting protocol for $n \geq 2$ players is envy-free (or completely fair) exactly if the degree of guaranteed envy-freeness equals $n(n-1)$.

Our next result shows the DGEF for a number of well-known finite bounded proportional cakecutting protocols 9 For the sake of self-containment, these protocols will be described in Section 5 ,

Theorem 3.5 For $n \geq 3$ players, 10 the proportional cake-cutting protocols listed in Table 1 have a degree of guaranteed envy-freeness as shown in the same table.

The proof of Theorem 3.5 can be found in Section 5. In particular, the proofs of Lemmas 5.1 through 5.6 provide the details of the analysis yielding the values in the DGEF column of Table 1 .

\begin{tabular}{|c|c|c|}
\hline Protocol & DGEF & Established via \\
\hline Last Diminisher [Ste48] & $2+n(n-1) / 2$ & Lemma 5.1 \\
\hline Lone Chooser [Fin64] & $n$ & Lemma 5.2 \\
\hline Lone Divider [Kuh67] & $2 n-2$ & Lemma 5.3 \\
\hline Cut Your Own Piece (no strategy) [Ste69] & $n$ & Lemma 5.4 \\
\hline Cut Your Own Piece (left-right strategy) & $2 n-2$ & Lemma 5.4 \\
\hline Divide and Conquer [EP84] & $n \cdot\lfloor\log n\rfloor+2 n-2^{\lfloor\log n\rfloor+1}$ & Lemma 5.5 \\
\hline Minimal-Envy Divide and Conquer [BJK07] & $n \cdot\lfloor\log n\rfloor+2 n-2^{\lfloor\log n\rfloor+1}$ & Lemma 5.5 \\
\hline Recursive Divide and Choose [Tas03] & $n$ & Lemma 5.6 \\
\hline
\end{tabular}

Table 1: DGEF of selected finite bounded cake-cutting protocols.

Apparently, the degrees of guaranteed envy-freeness of the protocols listed in Table 1 vary considerably. Although each of the protocols may provide an envy-free division in the best case, in the worst case some of them show just the minimum number of guaranteed envy-free-relations according to Proposition 3.3, while others possess a significantly higher degree of guaranteed envyfreeness. These differences can be explained by the fact that these protocols have been developed with a focus on achieving proportionality, and not on maximizing the degree of guaranteed envyfreeness. However, this indicates a new direction for future research, namely to increase the number of guaranteed envy-free-relations while ensuring finite boundedness. In the next section, we take a first step in this direction.

\footnotetext{
${ }^{9}$ These protocols may also be known under different names.

${ }^{10}$ The trivial cases $n=1$ (where one player receives all the cake) and $n=2$ (where each proportional division is always envy-free) are ignored. Specifically, an envy-free (and thus proportional) division for $n=2$ players can always be obtained by applying the cut-and-choose protocol: One player cuts the cake into two pieces both of which he or she considers to be worth exactly one half of the cake, and the other player chooses the piece that he or she considers to be worth at least half of the cake.
} 


\section{Enhancing the Degree of Guaranteed Envy-Freeness}

In this section, we introduce a finite bounded cake-cutting protocol that, compared with the protocols in Table 11 improves upon the degree of guaranteed envy-freeness. We will prove that this protocol is proportional and strategy-proof, and that it can be adapted so as to even provide a strong fair division. To present the protocol and its properties in an accessible way, we first handle the case of $n=4$ players separately in Section 4.1, before presenting and analyzing it for arbitrary $n \geq 3$ in Section 4.2

\subsection{A Proportional Protocol with an Enhanced DGEF for Four Players}

Figure 1 gives a finite bounded proportional cake-cutting protocol for four players. We give both the rules and the strategies at once. Note that the players have to follow the rules and strategies in order to obtain a proportional share of the cake in any case.

The protocol in Figure 1 always provides a proportional division (see Theorem 4.1) and has ten guaranteed envy-free-relations (see Theorem 4.2). To allow comparison for four players, the best DGEF of any of the proportional protocols listed in Table 1 (see also Section 5) - namely, that of both the Divide and Conquer protocols and the Last Diminisher protocol-is eight. Note that a maximum number of twelve guaranteed envy-free-relations is possible for four players (and this would give envy-freeness). Moreover, the protocol can be proven to be strategy-proof (see Theorem 4.4), and to even yield a strong fair division, provided that exactly one player makes a mark in Step 1 of the protocol that is closest to 1 (see Theorem 4.5).

Steps 4 through 12 of the protocol in Figure 1 (which is the part of the protocol when there are just three players left) is simply the Selfridge-Conway protocol 11 We explicitly describe the Selfridge-Conway protocol here for the sake of self-containment. Note that the Selfridge-Conway protocol is also part of the more involved protocol for an arbitrary number of players, which will be presented as Figure 3 in Section 4.2 .

Theorem 4.1 The cake-cutting protocol in Figure 1 is proportional.

Proof. Since all three players entering Step 4 consider the portion of the fourth player (who dropped out in Step 3) as being worth no more than 1/4, the Selfridge-Conway protocol is applied to a part of the cake that all three involved players consider as being worth at least $3 / 4$. Thus, since the Selfridge-Conway protocol is an envy-free (hence, in particular, a proportional) protocol, it by definition guarantees each of the three players entering Step 4 a portion of value at least 1/4 according to their measures. Moreover, the player who dropped out in Step 3 considers his or her portion to be worth $1 / 4$ due to Step 1 . Therefore, the cake-cutting protocol in Figure 1 always provides a proportional division for $n=4$ players.

Theorem 4.2 The cake-cutting protocol in Figure 1 has ten guaranteed envy-free-relations.

\footnotetext{
${ }^{11}$ The Selfridge-Conway protocol is known to be a finite bounded envy-free cake-cutting protocol for $n=3$ players (see Stromquist [Str80] and also, e.g., [BT95, BT96, RW98]).
} 


\begin{tabular}{|c|c|}
\hline Input: & $\begin{array}{l}\text { Cake } C \text {, and four players } p_{1}, p_{2}, p_{3}, p_{4} \text {, where } p_{i} \text { has the valuation function } v_{i} \text {. } \\
\text { Note that the value of cake } C \text { is normalized such that } v_{i}(C)=1,1 \leq i \leq 4 \text {. }\end{array}$ \\
\hline Output: & Mapping of portions $C_{i}$ to players $p_{i}$, where $C=\bigcup_{i=1}^{4} C_{i}$ \\
\hline Step 1. & Let each player $p_{i}, 1 \leq i \leq 4$, make a mark at $m_{i} \in C$, such that $v_{i}\left(m_{i}, 1\right)=1 / 4$. \\
\hline Step 2. & $\begin{array}{l}\text { Find any player } p_{j} \text { such that there is no player } p_{k}, 1 \leq j, k \leq 4, j \neq k \text {, with } \\
\left\|\left[m_{k}, 1\right]\right\|<\left\|\left[m_{j}, 1\right]\right\| . \text { (Ties can be broken arbitrarily.) }\end{array}$ \\
\hline Step 3. & Assign portion $C_{j}=\left[m_{j}, 1\right]$ to player $p_{j}$ and let $p_{j}$ drop out. \\
\hline \multicolumn{2}{|c|}{$\begin{array}{l}\text { Denote the remaining players by } p_{1}, p_{2} \text {, and } p_{3} \text {, without loss of generality, and let } v_{i} \text { be } \\
p_{i} \text { 's valuation function. }\end{array}$} \\
\hline Step 4. & $\begin{array}{l}\text { Let player } p_{1} \text { cut }\left[0, m_{j}\right] \text { into three pieces, say } c_{x}, c_{y} \text {, and } c_{z} \text {, of equal value } \\
\text { according to } v_{1} \text {. }\end{array}$ \\
\hline Step 5. & $\begin{array}{l}\text { If } v_{2}\left(c_{x}\right)>v_{2}\left(c_{y}\right) \text { and } v_{2}\left(c_{x}\right)>v_{2}\left(c_{z}\right),\{x, y, z\}=\{1,2,3\} \text {, let player } p_{2} \text { trim } \\
\text { piece } c_{x} \text { into piece } c_{x}^{\prime} \text { and trimmings } R \text { such that } v_{2}\left(c_{x}^{\prime}\right)=v_{2}\left(c_{y}\right) \geq v_{2}\left(c_{z}\right) \text { or } \\
v_{2}\left(c_{x}^{\prime}\right)=v_{2}\left(c_{z}\right) \geq v_{2}\left(c_{y}\right) \text {. If there already exists a two-way tie for the most } \\
\text { valuable piece according to } v_{2} \text {, do nothing. }\end{array}$ \\
\hline Step 6. & $\begin{array}{l}\text { Let player } p_{3} \text { choose one of the three pieces } c_{x} \text { (respectively, } c_{x}^{\prime} \text { if } c_{x} \text { has been } \\
\text { trimmed), } c_{y} \text {, or } c_{z} \text { that is most valuable according to } v_{3} \text {. }\end{array}$ \\
\hline Step 7. & $\begin{array}{l}\text { Let player } p_{2} \text { choose one piece from the two remaining pieces that is most } \\
\text { valuable according to } v_{2} \text {. If } c_{x}^{\prime} \text { is among the remaining pieces, player } p_{2} \text { has } \\
\text { to choose this one. }\end{array}$ \\
\hline Step 8. & Assign the remaining piece to player $p_{1}$ \\
\hline \multicolumn{2}{|c|}{$\begin{array}{l}\text { Step 8. Assign the remaining piece to player } p_{1} \text {. } \\
\text { If trimmings have been made in Step 5, continue with Step 9, otherwise finished. }\end{array}$} \\
\hline Step 9. & $\begin{array}{l}\text { Either player } p_{2} \text { or player } p_{3} \text { received the trimmed piece } c_{x}^{\prime} \text {. From these two, } \\
\text { let the player not having received } c_{x}^{\prime} \text { cut } R \text { into three equal pieces according } \\
\text { to his or her measure. }\end{array}$ \\
\hline Step 10. & $\begin{array}{l}\text { Let the player having received } c_{x}^{\prime} \text { choose one of these three pieces that is most } \\
\text { valuable according to his or her measure. }\end{array}$ \\
\hline Step 11. & $\begin{array}{l}\text { Let player } p_{1} \text { choose one out of the two remaining pieces that is most valuable } \\
\text { according to } v_{1} \text {. }\end{array}$ \\
\hline Step 12. & Assign the remaining piece to the player that cut $R$. \\
\hline
\end{tabular}

Figure 1: A finite bounded proportional cake-cutting protocol with DGEF of 10 for four players.

Proof. The DGEF of the protocol in Figure 1 can be justified analogously to the arguments in the proof of Theorem 4.1. Because the Selfridge-Conway protocol always provides an envy-free division, there is no envy between the three players entering Step 4, which results in six guaranteed envy-free-relations. In addition, the same three players will not envy the player (call him or her $p_{j}$ ) who dropped out in Step 3 with portion $C_{j}$, since none of them valued portion $C_{j}$ as being worth more than $1 / 4$ and each of them is guaranteed a share of at least $(1 / 3)(3 / 4)=1 / 4$, which gives three more guaranteed envy-free-relations. Simply put, none of the three players entering Step 4 will envy any of the three other players, summing up to nine guaranteed envy-free-relations. By the argument in the proof of Proposition 3.3, no more envy-free-relations can be guaranteed on behalf of these 
three players. The last guaranteed envy-free-relation is due to player $p_{j}$ : Since the protocol always provides a proportional division, $p_{j}$ cannot envy each of the three remaining players (again by the proof of Proposition 3.3). However, it cannot be guaranteed that $p_{j}$ does not envy any of the other two remaining players either, since $p_{j}$ does not evaluate their portions. This follows from the proof of Lemma 3.4$]^{12}$ Thus, the protocol shown in Figure 1 has a DGEF of exactly ten in total.

Thinking of manipulation aspects, there may be players who try to gain most of the cake for themselves, or who intentionally try to make other players envious. To prevent this from happening, cake-cutting protocols should be strategy-proof.

Definition 4.3 A proportional cake-cutting protocol is said to be strategy-proof if a cheating player is no longer guaranteed a proportional share, whereas all other players are still guaranteed to receive their proportional share.

In a strategy-proof proportional protocol, a cheater (i.e., a player who doesn't play truthfully) cannot harm any of the other players with respect to proportionality, and may even jeopardize receiving a proportional share of the cake for him- or herself.

It is worth noting that the definition of strategy-proofness is slightly stronger when restricted to envy-free cake-cutting protocols. An envy-free cake-cutting protocol is said to be strategy-proof if a cheating player is no longer guaranteed to not envy any other player, whereas all other players are. That is, a strategy-proof envy-free cake-cutting protocol is resistant to manipulation in the sense that for a player to be guaranteed to not envy any other player, he or she is required to play truthfully.

Theorem 4.4 The proportional cake-cutting protocol in Figure $\square$ is strategy-proof in the sense of Definition 4.3

Proof. When analyzing the strategy-proofness of the protocol in Figure 1, only decisions made in Steps 1 through 3 need to be considered, since the Selfridge-Conway protocol has already been proven to be strategy-proof (see, e.g., [BT96]). So, in each of the following three cases, we thus will consider only Steps 1 through 3 of the protocol. Moreover, it is assumed that there is exactly one cheater (i.e., one player not playing truthfully) that is trying to get more than a proportional share, call him or her $p_{c}, 1 \leq c \leq 4$.

Case 1: If $p_{c}$ is the player to drop out in Step 3 with portion $C_{c}=\left[m_{c}, 1\right], v_{c}\left(m_{c}, 1\right)>1 / 4$, then the cheater would receive more than a proportional share according to his or her measure. However, all other players are still guaranteed a proportional share, since all of them consider portion $C_{c}$ as being worth at most $1 / 4$ according to their measures, so they all consider the remaining part of the cake to be worth at least $3 / 4$.

\footnotetext{
${ }^{12}$ More specifically, consider the following scenario. Suppose the fourth player dropped out in Step 3 (so $j=4$ ), and $p_{4}$ values his or her portion $C_{4}$ to be worth exactly $1 / 4$ and the remaining cake $\left[0, m_{4}\right]$ to be worth exactly $3 / 4$. If we set the valuation functions such that $v_{1}\left(C_{1}\right)=v_{2}\left(C_{2}\right)=v_{3}\left(C_{3}\right)=1 / 4, v_{4}\left(C_{1}\right)=1 / 8$, and $v_{4}\left(C_{2}\right)=v_{4}\left(C_{3}\right)=5 / 16$ (thus, $\left.v_{4}\left(C_{1}\right)+v_{4}\left(C_{2}\right)+v_{4}\left(C_{3}\right)=3 / 4\right)$, dividing cake $C$ into $C_{1}, C_{2}, C_{3}$, and $C_{4}$ results in a proportional division such that player $p_{4}$ envies players $p_{2}$ and $p_{3}$.
} 
Case 2: If the cheater, $p_{c}$, would have dropped out in Step 3 with portion $C_{c}=\left[m_{c}, 1\right]$ when telling the truth (i.e., $v_{c}\left(m_{c}, 1\right)=1 / 4$ and $v_{i}\left(m_{c}, 1\right) \leq 1 / 4$ with $1 \leq i \leq 4$ and $i \neq c$ ) but now is not (since $p_{c}$ makes a mark at $m_{c}^{\prime}$ with $m_{c}^{\prime}<m_{c}$ ), then the cheater may end up with even less than $1 / 4$. Let us see why this is true. In this case, another player, $p_{j}$, drops out in Step 3 with portion $C_{j}=\left[m_{j}, 1\right]$, where $m_{c}^{\prime} \leq m_{j} \leq m_{c}$, which determines the subpart of the cake for Step 4 and the following steps to be $\left[0, m_{j}\right]$. According to the measure of the cheater, subpart $\left[0, m_{j}\right]$ is worth at most $3 / 4$, since he or she values $\left[m_{j}, 1\right]$ as being worth at least $1 / 4$, as assumed beforehand. Applying the Selfridge-Conway protocol to subpart $\left[0, m_{j}\right]$ guarantees each of the involved players at least a proportional share, which results for the cheater in a portion that may be worth even less than $(1 / 3)(3 / 4)=1 / 4$ (namely, if $m_{j}<m_{c}$ ). (Note that this is the point where the cheater loses his or her guarantee for a proportional share via cheating.)

Again, all other players are still guaranteed a proportional share. The player receiving portion $C_{j}=\left[m_{j}, 1\right]$ values this portion as being $1 / 4$. The two remaining players both value subpart $\left[0, m_{j}\right]$ to be worth at least $3 / 4$, and thus receive a portion that is worth at least $(1 / 3)(3 / 4)$ according to their measures.

Case 3: If the cheater does not drop out in Step 3 by cheating, nor would have dropped out in Step 3 if he or she would have been truthfully, then this would not influence the division at all. The player dropping out in Step 3 with portion $C_{j}=\left[m_{j}, 1\right]$ values this portion as being $1 / 4$, and the remaining players receive a proportional share of subpart $\left[0, m_{j}\right]$, which all of them value at least $3 / 4$, even the cheater.

This concludes the proof of Theorem 4.4 .

Moreover, just a little change in the procedure can make the protocol presented above to provide not just a simple fair but a strong fair division for four players, which means that every player considers the portion received to be worth strictly more than one quarter of $C$.

Theorem 4.5 The cake-cutting protocol in Figure 1 can be modified so as to yield a strong fair division, provided that exactly one player makes a mark in Step 1 that is closest to 1 (with respect to the interval $[0,1])$.

Proof. Figure 2 shows only the modified steps of the protocol in Figure 11 that are required to achieve a strong fair division.

Let $p_{j}$ be the unique player whose mark in Step 1 is closest to 1 . According to Step 2 in Figure2, let $p_{k}$ be any player such that $\left\|\left[m_{j}, 1\right]\right\|<\left\|\left[m_{k}, 1\right]\right\|$ and there is no player $p_{\ell}, 1 \leq j, k, \ell \leq 4$, $\|\{j, k, \ell\}\|=3$, with $\left\|\left[m_{\ell}, 1\right]\right\|<\left\|\left[m_{k}, 1\right]\right\|$, where ties can be broken arbitrarily. Step 3 in Figure 2 assures that player $p_{i}$ is dropping out with a portion that is worth strictly more than $1 / 4$ according to his or her measure 13 since $p_{j}$ receives a portion that is bigger and thus is worth more than the one he or she has marked as being worth exactly $1 / 4$. The three remaining players continue by applying a proportional protocol to a part of the cake that all of them consider to be worth strictly more than $3 / 4$.

\footnotetext{
${ }^{13}$ Recall that we assumed the axiom of positivity, which requires nonempty pieces of cake to have a nonzero value for each player, see also Footnote 3
} 
Thus, each of the players receives a portion that is worth strictly more than $1 / 4$ according to his or her measure, which results in a strong fair division.

\begin{tabular}{|l}
\hline Step 2. Find any players $p_{j}$ and $p_{k}$ such that $\left\|\left[m_{j}, 1\right]\right\|<\left\|\left[m_{k}, 1\right]\right\|$ and there is no \\
player $p_{\ell}, 1 \leq j, k, \ell \leq 4,\|\{j, k, \ell\}\|=3$, with $\left\|\left[m_{\ell}, 1\right]\right\|<\left\|\left[m_{k}, 1\right]\right\|$. (Ties \\
can be broken arbitrarily.) \\
Step 3. Set $m=m_{k}+\left(m_{j}-m_{k}\right) / 2$ and assign portion $C_{j}=[m, 1]$ to player $p_{j}$ and let $p_{j}$ \\
drop out. \\
Denote the remaining players by $p_{1}, p_{2}$, and $p_{3}$, without loss of generality, and let $v_{i}$ be \\
$p_{i}$ 's valuation function. \\
Step 4. Let player $p_{1}$ cut $[0, m]$ into three pieces, say $c_{x}, c_{y}$ and $c_{z}$, of equal value \\
according to $v_{1}$.
\end{tabular}

Figure 2: Modified steps in the protocol of Figure 1 to achieve a strong fair division for four players.

\subsection{A Proportional Protocol with an Enhanced DGEF for any Number of Players}

Figure 3 shows a finite bounded proportional cake-cutting protocol with an enhanced DGEF for $n$ players, where $n \geq 3$ is arbitrary. Again, we give both the rules and the strategies at once, and players have to follow the rules and strategies in order to obtain a proportional share of the cake. Unless specified otherwise, ties in this protocol can be broken arbitrarily. With respect to the DGEF results of previously known finite bounded proportional cake-cutting protocols given in Table 1 (see also Section [5), the Last Diminisher protoco 14 shows the best results for $n \geq 6$, whereas the best results for $n<6$ are achieved by the Last Diminisher protocol as well as both the Divide and Conquer protocols [EP84, BJK07]. The protocol presented in Figure 3 improves upon these protocols in terms of the degree of guaranteed envy-freeness for all $n \geq 3$ and, in particular, improves upon the DGEF of the Last Diminisher protocol by $\lceil n / 2\rceil-1$ additional guaranteed envy-free-relations 15

Both the protocol in Figure 3 and the Last Diminisher protocol are, more or less, based on the same idea of determining a piece of minimal size that is valued exactly $1 / n$ by one of the players (who is still in the game), which guarantees that all other players (who are still in the game) will not envy this player for receiving this particular piece. However, the protocol in Figure 3 works in a more parallel way, which makes its enhanced DGEF of $\left[n^{2} / 2\right]+1$ possible (see Theorem 4.9), and it forbears from using trimmings. To ensure that working in a parallel manner indeed pays off in terms of increasing the degree of guaranteed envy-freeness, the "inner loop" (Steps 4.1 through 4.3) of the protocol is decisive.

In addition, the protocol in Figure 3 always provides a proportional division (see Theorem 4.7) in a finite bounded number of steps (see Theorem 4.8), it can be proven to be strategy-proof (in the sense of Definition 4.3, see Theorem 4.10), and analogously to the modification described in

\footnotetext{
${ }^{14}$ This protocol has been developed by Banach and Knaster and was first presented in Steinhaus [Ste48].

${ }^{15}$ Recall that we ignore the trivial cases $n=1$ and $n=2$, see Footnote 10 Moreover, in the special case of $n=4$ the DGEF of the protocol in Figure 3 is $\left(n^{2} / 2\right)+2=10$ and thus improves the DGEF of the Last Diminisher protocol by even $n / 2=2$ guaranteed envy-free-relations, see Theorem 4.2
} 
Section 4.1, this protocol can be adjusted to provide a strong fair division for suitable valuation functions of the players (see Theorem 4.11). All of the above properties are shared also by the Last Diminisher protocol (see, e.g., [RW98]).

Remark 4.6 Some remarks on the protocol in Figure 3 are in order:

1. From a very high-level perspective the procedure is as follows: The protocol runs over several rounds in each of which it is to find a player $p_{j}$ who takes a portion from the left side of the cake, and to find a player $p_{k}$ who takes a disjoint portion from the right side of the cake, such that none of the players still in the game envy $p_{j}$ or $p_{k}$ (at this, appropriate "inner-loop handling" might be necessary, see Figure 3 for details). Thereafter, $p_{j}$ and $p_{k}$ are to drop out with their portions, and a new round is started with the remaining cake (which is being renormalized, see Remarks 3 and 5 below) and the remaining players. Finally, the SelfridgeConway protocol is applied to the last three players in the game.

2. Note that this protocol is applied only if there are more than two players in total. If there is just one player then he or she receives all the cake, and if there are only two players then the simple cut-and-choose protocol is applied (see Footnote 10).

3. Regarding $n \geq 5$ players, if at any stage of our protocol the same player marks both the leftmost smallest piece and the rightmost smallest piece, the cake may be split up into two pieces and later on merged again. To simplify matters, in such a case the interval boundaries are adapted as well, which is expressed in Step 8 of Figure 3 Simply put, the two parts of the cake are set next to each other again to ensure a seamless transition. This can be done without any loss in value due to additivity of the players' valuation functions.

4. Note that if the inner loop (Steps 4.1 through 4.3) has not been executed in an outer-loop iteration (Steps 1 through 8), we have $\rho=\rho^{\prime}$. This is the special case of zero iterations of the inner loop. Consequently, if $\rho=\rho^{\prime}$ then the portion $C_{k}=\left[\rho_{k}, \rho\right]$ assigned to player $p_{k}$ in Step 6 is the same as $C_{k}=\left[\rho_{k}, \rho^{\prime}\right]$ in the general case, and the values for $\rho:=\rho_{k}$ and $C^{\prime}:=\left[\lambda_{j}, \rho_{k}\right]$ that are set in Step 8 in this case are special cases of $\rho:=\rho-\rho^{\prime}+\rho_{k}$ and $C^{\prime}:=\left[\lambda_{j}, \rho_{k}\right] \cup\left[\rho^{\prime}, \rho\right]$ (since if $\rho=\rho^{\prime}$ then $\left[\rho^{\prime}, \rho\right]$ degenerates to a single point, which is valued zero by the axiom of divisibility, see Footnote (4). However, to make the protocol in Figure 3 easier to comprehend, we have stated these special cases explicitly in addition to the general case.

5. In Steps 1 and 9.1, the value of subcake $C^{\prime} \subseteq C$ is renormalized such that $v_{i}\left(C^{\prime}\right)=1$ for each player $p_{i}, 1 \leq i \leq s$, for the sake of convenience. In more detail, each player $p_{i}$ values $C^{\prime}$ at least $s / n$ of $C$, i.e., $v_{i}\left(C^{\prime}\right) \geq(s / n) \cdot v_{i}(C)$. The latter holds true since each of the $s$ players still in the game values the union of the $n-s$ portions already assigned to be worth at most $(n-s) / n$ of $C$. Thus, by receiving a proportional share (valued $1 / s$ ) of $C^{\prime}$ each player $p_{i}$ is guaranteed at least a proportional share (valued $1 / n$ ) of $C$.

6. Note that Steps 9.1 through 9.3 of the protocol in Figure 3 correspond to Steps 1 through 3 of the protocol in Figure $\square$ and that Steps 9.1 through 9.4 are performed exactly if the initial number $n$ of players is even. 


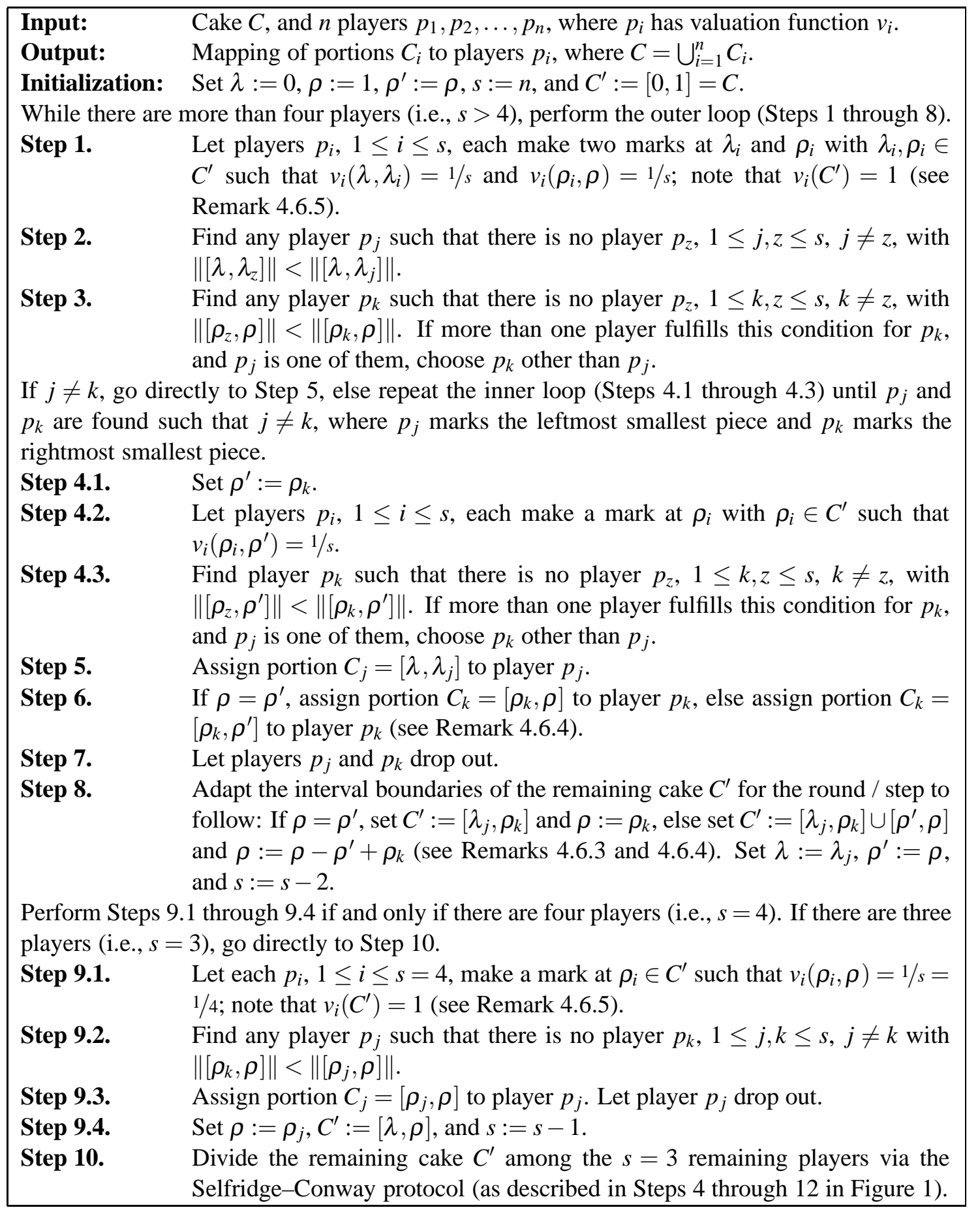

Figure 3: A proportional protocol with an enhanced DGEF of $\left\lceil n^{2} / 2\right\rceil+1$ for $n \geq 3$ players. 


\section{Theorem 4.7 The cake-cutting protocol in Figure 3 is proportional.}

Proof. In the case of $n$ being even, all four players entering Step 9.1 consider $C^{\prime}$ at this stage to be worth at least $s / n=4 / n$ of $C$, since each of them values the union of the $n-4$ portions already assigned to be worth at most $(n-4) / n$ of $C$. The same argument can be applied to the three players that enter Step 10 in the case of $n$ being odd. Thus, from Step 9.1 on, which is the part when there are no more than four players left, the protocol provides a proportional division according to Theorem 4.1 .

When there are more than four players in total, either the first $n-4$ players (if $n$ is even), or the first $n-3$ players (if $n$ is odd), receive a portion they each value to be worth at least $1 / n$ according to Steps 1 and 4.2, since each of these players receives a portion he or she once specified to be worth exactly $1 / s$ of $C^{\prime}$, while valuing $C^{\prime}$ at least $s / n$ of $C$ (see Remark 4.6[5).

Thus, the cake-cutting protocol in Figure 3 always provides a proportional division for any number $n \geq 3$ of players.

\section{Theorem 4.8 The cake-cutting protocol in Figure 3 is finite bounded.}

Proof. The protocol in Figure 3 has only a finite number of steps-with two loops though. The outer loop (which repeats Steps 1 through 8 as long as there are more than either four players if $n$ is even, or three players if $n$ is odd) is iterated $(n-4) / 2$ times if $n$ is even, and is iterated $(n-3) / 2$ times if $n$ is odd. The inner loop (which repeats Steps 4.1 through 4.3 until two distinct players are found to which the two outermost pieces are assigned, one player receiving that from the present left and the other player receiving that from the present right boundary) is iterated at most $s-2$ times per outer-loop iteration with $s$ players, summing up to at most $\sum_{i=1}^{[(n-4) / 2\rceil}(n-2 i)$ iterations of the inner loop in total. Let us see why this is true. For each outer-loop iteration with $s$ players, the inner loop is iterated as long as the player that marked the leftmost smallest piece also marks the rightmost smallest piece (with respect to the current right boundary $\rho^{\prime}$ ) and there is no tie with another player for the rightmost smallest piece. With respect to every single player $p_{i}, 1 \leq i \leq s$, when setting $v_{i}\left(C^{\prime}\right)=1$, the division of $C^{\prime}$ into pieces valued $1 / s$ each results in $s$ disjoint pieces. Two of these $s$ pieces have been identified in Steps 2 and 3 already and thus only $s-2$ more pieces can be identified. At this, let $p_{j}$ be the player that is to be assigned the leftmost smallest piece $\left[\lambda, \lambda_{j}\right]$ in Step 5 and the only player that marked the rightmost smallest piece $\left[\rho_{j}, \rho\right]$. Then there must be a player $p_{k}, k \neq j$, with $v_{k}\left(\lambda, \lambda_{j}\right) \leq 1 / s$ and $v_{k}\left(\rho_{j}, \rho\right)<1 / s$, and there is at least one piece $\left[\rho_{k}, \rho^{\prime}\right]$ among the $s-2$ remaining pieces for which it is true that $v_{k}\left(\rho_{k}, \rho^{\prime}\right)=1 / s$ and $v_{j}\left(\rho_{k}, \rho^{\prime}\right) \leq 1 / s$, i.e., for some $\rho^{\prime}$ it holds that $\left\|\left[\rho_{k}, \rho^{\prime}\right]\right\| \leq\left\|\left[\rho_{j}, \rho^{\prime}\right]\right\|$. Hence, within any outer-loop iteration, the inner loop stops after at most $s-2$ iterations. Counting in single steps, Steps 1, 2, 3, 5, 6, 7, and 8 each are repeated $\lceil(n-4) / 2\rceil$ times, Steps $4.1,4.2$ and 4.3 each are repeated at most $\sum_{i=1}^{\lceil(n-4) / 2\rceil}(n-2 i)$ times, Steps 9.1 through 9.4 each are repeated at most once, and Step 10 involves at most nine more steps (according to Figure 1). Thus, in terms of single steps as presented in Figure 3, the protocol is bounded by $(7 \cdot\lceil(n-4) / 2\rceil)+\left(3 \cdot \sum_{i=1}^{\lceil(n-4) / 2\rceil}(n-2 i)\right)+4+9$ steps. Thus, the protocol in Figure 3 carries out only finitely many operations and is finite bounded. 
Theorem 4.9 For $n \geq 5$ players, the cake-cutting protocol in Figure 3 has $\left\lceil n^{2} / 2\right\rceil+1$ guaranteed envy-free-relations. 16

Proof. The DGEF of the protocol in Figure 3 increases every time a portion is assigned to a player. Considering Steps 1 through 8 of any outer-loop iteration with $s$ players, player $p_{j}$ (the player receiving the leftmost smallest piece in Step 5 and dropping out with this portion in Step 7) will not be envied by any of the $s-1$ other players. Regarding player $p_{k}$ (the player receiving the rightmost smallest piece in Step 6 and dropping out with this portion in Step 7), two cases need to be considered - the best case and the worst case. In the best case, player $p_{k}$ immediately is not the same one as player $p_{j}$. In the worst case, players $p_{j}$ and $p_{k}$ are one and the same player (i.e., $j=k$ ) in Step 3, and Steps 4.1 through 4.3 need to be executed. In this case, as already mentioned in the proof of Theorem 4.8, there must be at least one piece $\left[\rho_{k}, \rho^{\prime}\right]$ with $v_{k}\left(\rho_{k}, \rho^{\prime}\right)=1 / s$ and $v_{j}\left(\rho_{k}, \rho^{\prime}\right) \leq 1 / s$, i.e., for some $\rho^{\prime}$ it holds that $\left\|\left[\rho_{k}, \rho^{\prime}\right]\right\| \leq\left\|\left[\rho_{j}, \rho^{\prime}\right]\right\|$. Consequently, in both the best and the worst case the player receiving the rightmost smallest piece (with respect to the current right boundary, either $\rho$ or some $\rho^{\prime}$ ) will not be envied by any of the $s-1$ other players, not even by player $p_{j}$. However, none of the players $p_{j}$ and $p_{k}$ can be guaranteed to be not envied by more than $s-1$ players according to the proof of Lemma 3.4 .

Since the outer loop is repeated $\lceil(n-4) / 2\rceil$ times, the number of guaranteed envy-free-relations among the players sums up to $\left(n^{2} / 2\right)-8$ when $n$ is even, and to $\left\lfloor n^{2} / 2\right\rfloor-4=\left\lceil n^{2} / 2\right\rceil-5$ when $n$ is odd. Note that $s$ is decreasing in every outer-loop iteration, and so $1 / s$ is increasing, which implies that no player receiving a portion valued $1 / s$ at a later outer-loop iteration will envy any of those players that received a portion in one of the previous outer-loop iterations. To verify the latter, let us denote by $C_{t}^{\prime}$ the subcake to be divided and by $s_{t}$ the number of players participating in outerloop iteration $t$ with $2 \leq t \leq\lceil(n-4) / 2\rceil$. Considering any outer-loop iteration $t-1$ with cake $C_{t-1}^{\prime}$ being renormalized such that $v_{i}\left(C_{t-1}^{\prime}\right)=1,1 \leq i \leq s_{t-1}$, each of the two players dropping out in iteration $t-1$ receives a portion he or she values exactly $1 / s_{t-1}$ of $C_{t-1}^{\prime}$ and which is valued at most $1 / s_{t-1}$ of $C_{t-1}^{\prime}$ by the remaining players, where $v_{i}\left(C_{t-1}^{\prime}\right) \geq s_{t-1} / n$ (see Remark 4.615). Consequently, all players $p_{j}, 1 \leq j \leq s_{t}$, participating in outer-loop iteration $t$ value subcake $C_{t}^{\prime}$ to be worth at least $s_{t} / s_{t-1}$ of $C_{t-1}^{\prime}$, i.e., $v_{j}\left(C_{t}^{\prime}\right) \geq\left(s_{t} / s_{t-1}\right) \cdot v_{j}\left(C_{t-1}^{\prime}\right)$, and the two players dropping out in iteration $t$ both receive a portion valued $1 / s_{t}$ of subcake $C_{t}^{\prime}$. Thus, players dropping out in outer-loop iteration $t$ receive each a portion that is valued at least $1 / s_{t-1}$ of $C_{t-1}^{\prime}$ according to their measures.

Once the outer loop has been completed, either four (if $n$ is even) or three (if $n$ is odd) players are left. If there are four players left, three more envy-free-relations can be guaranteed by executing Steps 9.1 through 9.4, as the three remaining players will not envy the player receiving the fourthto-last portion, but no more than three envy-free-relations can be guaranteed, again by the proof of Lemma 3.4. In addition, the three last players entering Step 10 will not envy each other, because the Selfridge-Conway protocol always provides an envy-free division. Accordingly, six more envyfree-relations are guaranteed. Summing up, the protocol in Figure 3 has a DGEF of $\left\lceil n^{2} / 2\right\rceil+1$.

Theorem 4.10 The proportional cake-cutting protocol in Figure 3 is strategy-proof in the sense of Definition 4.3

\footnotetext{
${ }^{16}$ Note that the same formula holds if $n=3$, but for the special case of $n=4$ even one more envy-free-relation can be guaranteed (see Theorem 4.2).
} 
Proof. From Step 9.1 on (when at most four players are left), the protocol has been proven to be strategy-proof in Theorem 4.4. With respect to Steps 1 through 8, three different cases need to be considered, each of which applies analogously to both assigning the leftmost smallest piece and assigning the rightmost smallest piece. Note that a cheating attempt on one of the pieces does not influence the assignment of the other. The same three cases apply when there is a cheating attempt on the rightmost smallest piece and Steps 4.1 through 4.3 need to be executed. In the following case distinction, given any iteration $t$ of the outer loop and cake $C_{t}^{\prime}=[\lambda, \rho]$ with $v_{i}\left(C_{t}^{\prime}\right)=1$ for all players $p_{i}, 1 \leq i \leq s_{t}$, we will consider only the situation when there is exactly one player not telling the truth with respect to the leftmost smallest piece, trying to get more than a proportional share. Let $p_{c}$ be this cheating player, where $1 \leq c \leq s_{t}$.

Case 1: If $p_{c}$ is the player receiving portion $C_{c}=\left[\lambda, \lambda_{c}\right]$ with $v_{c}\left(\boldsymbol{\lambda}, \boldsymbol{\lambda}_{c}\right)>1 / s_{t}$ in Step 5 (and dropping out in Step 7), then $p_{c}$ would receive more than a proportional share. However, all $s_{t}-1$ other players are still guaranteed a proportional share, since all of them consider portion $C_{c}$ as being worth at most $1 / s_{t}$ of $C_{t}^{\prime}$ according to their measures, so they all consider the remaining part of the cake to be worth at least $\left(s_{t}-1\right) / s_{t}$ of $C_{t}^{\prime}$.

Case 2: If the cheater, $p_{c}$, would have received portion $C_{c}=\left[\lambda, \lambda_{c}\right]$ in Step 5 when telling the truth (so $v_{c}\left(\lambda, \lambda_{c}\right)=1 / s_{t}$ and $v_{i}\left(\lambda, \lambda_{c}\right) \leq 1 / s_{t}$ with $1 \leq i \leq s_{t}$ and $i \neq c$ ) but now is not (since $p_{c}$ is making a mark at $\lambda_{c}^{\prime}$ with $\lambda_{c}<\lambda_{c}^{\prime}$ ), then the cheater could end up with even less than a proportional share. Let us see why this holds true. In this case, another player, $p_{j}$ with $j \neq c$, receives portion $C_{j}=\left[\lambda, \lambda_{j}\right], \lambda_{c} \leq \lambda_{j} \leq \lambda_{c}^{\prime}$, which determines the left boundary of $C_{t+1}^{\prime}$ (the remaining cake to be continued with) to be $\lambda_{j}$. According to the measure of player $p_{c}$, $\left[\lambda_{j}, \rho\right]$ is worth at most $\left(s_{t}-1\right) / s_{t}$ of $C_{t}^{\prime}$, because he or she values $\left[\lambda, \lambda_{j}\right]$ to be worth at least $1 / s_{t}$ of $C_{t}^{\prime}$, as assumed beforehand.

However, since the protocol works in a parallel way, this loss in value may be compensated for by some gain in value with respect to the rightmost smallest piece $C_{k}=\left[\rho_{k}, \rho^{\prime \prime}\right]$ that is assigned in Step 6 (where $\rho_{k}$ is the left boundary of the rightmost smallest piece marked by some player $p_{k},\|\{c, j, k\}\|=3$, and $\rho^{\prime \prime}$ is the current right boundary, i.e., either $\rho^{\prime \prime}=\rho$ or $\rho^{\prime \prime}=\rho^{\prime}$ ). That is, if $v_{c}\left(\lambda_{c}, \lambda_{j}\right) \leq v_{c}\left(\rho_{c}, \rho_{k}\right)$ then the loss player $p_{c}$ experiences by cheating with respect to the leftmost smallest piece is made up for by an accidentally sufficient gain relative to the assignment of the rightmost smallest piece 17 However, if $v_{c}\left(\lambda_{c}, \lambda_{j}\right)>v_{c}\left(\rho_{c}, \rho_{k}\right)$ then no sufficient compensation takes place and player $p_{c}$ considers $C_{t+1}^{\prime}=\left[\lambda_{j}, \rho_{k}\right]$, which is the part of the cake to be continued with and to be divided among $s_{t}-2$ players, as being worth at most $\left(s_{t}-2\right) / s_{t}$ of $C_{t}^{\prime}$. Thus, in this case player $p_{c}$ is not guaranteed a portion that is worth at least $1 / n$ of $C$ in total (according to his or her measure), even though the protocol would be proportional if all players were playing by the rules and strategies required by the protocol. Again, all other players are still guaranteed a proportional share. Players $p_{j}$ and $p_{k}$ both value their portion to be $1 / s_{t}$ of $C_{t}^{\prime}$, and the $s_{t}-3$ remaining players consider $C_{t+1}^{\prime}=\left[\lambda_{j}, \rho_{k}\right]$ (which is to be divided among $s_{t}-2$ players) as being worth at least $\left(s_{t}-2\right) / s_{t}$ of $C_{t}^{\prime}$.

\footnotetext{
${ }^{17}$ Note that the compensation for the cheater's loss may also be accumulated over the following rounds as long as the cheater has not been assigned a portion yet.
} 
Note that if the cheater marks the rightmost smallest piece in the very same outer-loop iteration in which he or she is cheating on the leftmost smallest piece and happens to receive the rightmost smallest piece, then there is no influence on the portions to be assigned in this outer-loop iteration, as even the cheater will receive a proportional share being worth $1 / s_{t}$ of $C_{t}^{\prime}$ according to his or her measure.

Case 3: If the cheater does not receive the leftmost smallest portion $C_{j}=\left[\lambda, \lambda_{j}\right]$ by cheating nor would have received this portion when telling the truth, then this would not at all affect the leftmost portion to be assigned in this particular outer-loop iteration. The player receiving portion $C_{j}=\left[\lambda, \lambda_{j}\right]$ in Step 5 values this portion as being $1 / s_{t}$ of $C_{t}^{\prime}$, and the remaining players (except for some player $p_{k}, j \neq k$, that is assigned the rightmost smallest piece) continue the procedure with $C_{t+1}^{\prime}=\left[\lambda_{j}, \rho_{k}\right]$ (where $\rho_{k}$ is the left boundary of the rightmost smallest piece marked by $p_{k}$ ), which each of them values at least $\left(s_{t}-2\right) / s_{t}$ of $C_{t}^{\prime}$, even the cheater.

This concludes the proof of Theorem 4.10 .

Figure 4 shows how to adapt the protocol in Figure 3 so as to achieve a strong fair division. Again, note that if the inner loop (Steps 4.1 through 4.3) has not been executed in an outer-loop iteration (Steps 1 through 8), we have the special case of zero inner-loop iterations and $\rho=\rho^{\prime}$. In this case, portion $C_{k}=\left[\rho_{k}-m_{r}, \rho\right]$ assigned to player $p_{k}$ in Step 6 is the same as $C_{k}=\left[\rho_{k}-m_{r}, \rho^{\prime}\right]$ in the general case, and the values for $\rho:=\rho_{k}-m_{r}$ and $C^{\prime}:=\left[\lambda_{j}+m_{\ell}, \rho_{k}-m_{r}\right]$ that are set in Step 8 in this case are special cases of $\rho:=\rho-\rho^{\prime}+\rho_{k}-m_{r}$ and $C^{\prime}:=\left[\lambda_{j}+m_{\ell}, \rho_{k}-m_{r}\right] \cup\left[\rho^{\prime}, \rho\right]$ (again, since $\left[\rho^{\prime}, \rho\right]$ degenerates to a single point if $\rho=\rho^{\prime}$, which is valued zero by the axiom of divisibility, see Footnote 4).

Theorem 4.11 For $n>3$ players, modifying the first iteration of the cake-cutting protocol in Figure 3 according to Figure 4 yields a strong fair division, provided that in this iteration exactly one player makes a mark that is closest to the left boundary and exactly one distinct player makes a mark that is closest to the right boundary.

Proof. For $n=4$ players, the protocol in Figure 3 is the same as the protocol in Figure 1 and thus can be modified so as to yield a strong fair division according to Theorem 4.5, see Figure 2 ,

For $n>4$ players, consider the very first outer-loop iteration (Steps 1 through 8 ) of the protocol. Let $p_{j}$ be the unique player whose mark in Step 1 is closest to the left boundary $\lambda$, and let $p_{k}, j \neq k$, be the unique player whose mark in Step 1 is closest to the right boundary $\rho$ (if the inner loop needs to be executed, let $p_{k}$ be the unique player whose mark in Step 4.2 is closest to the current right boundary $\left.\rho^{\prime}\right)$. According to Step 2 in Figure 4 , let $p_{\ell}$ be any player such that $\left\|\left[\lambda, \lambda_{j}\right]\right\|<\left\|\left[\lambda, \lambda_{\ell}\right]\right\|$ and there is no player $p_{z}, 1 \leq j, \ell, z \leq s,\|\{j, \ell, z\}\|=3$, with $\left\|\left[\lambda, \lambda_{z}\right]\right\|<\left\|\left[\lambda, \lambda_{\ell}\right]\right\|$, where ties can be broken arbitrarily. Analogously, according to Step 3 (respectively, Step 4.3) in Figure 4, let $p_{r}$ be any player such that $\left\|\left[\rho_{k}, \rho\right]\right\|<\left\|\left[\rho_{r}, \rho\right]\right\|$ (respectively, $\left\|\left[\rho_{k}, \rho^{\prime}\right]\right\|<\left\|\left[\rho_{r}, \rho^{\prime}\right]\right\|$ ) and there is no player $p_{z}, 1 \leq k, r, z \leq s,\|\{k, r, z\}\|=3$, with $\left\|\left[\rho_{z}, \rho\right]\right\|<\left\|\left[\rho_{r}, \rho\right]\right\|\left(\left\|\left[\rho_{z}, \rho^{\prime}\right]\right\|<\left\|\left[\rho_{r}, \rho^{\prime}\right]\right\|\right)$. Steps 5 and 6 in Figure 4 assure that players $p_{j}$ and $p_{k}$ each are assigned a portion that, according to their measures, is worth strictly more than $1 / s$ of $C^{\prime}$ (which is worth at least as much as $1 / n$ of $C$ by the 
argument of Remark 4.65, 18 since $p_{j}$ and $p_{k}$ each receive a portion that is bigger and thus is worth more than the one they have marked as being worth exactly $1 / s$ of $C^{\prime}$ in Step 1.

By the same argument, the $n-2$ remaining players continue by applying a proportional protocol to a part of the cake that all of them consider to be worth strictly more than $(n-2) / n$ of $C$. Thus, each of the players receives a portion that is worth strictly more than $1 / n$ of $C$ according to his or her measure, which results in a strong fair division.

\begin{tabular}{ll}
\hline Step 2. & Find any players $p_{j}$ and $p_{\ell}$ such that $\left\|\left[\lambda, \lambda_{j}\right]\right\|<\left\|\left[\lambda, \lambda_{\ell}\right]\right\|$ and there is no \\
& player $p_{z}, 1 \leq j, \ell, z \leq s,\|\{j, \ell, z\}\|=3$, with $\left\|\left[\lambda, \lambda_{z}\right]\right\|<\left\|\left[\lambda, \lambda_{\ell}\right]\right\|$. Set $m_{\ell}:=$ \\
& $\left(\lambda_{\ell}-\lambda_{j}\right) / 2$.
\end{tabular}

Step 3. Find any players $p_{k}$ and $p_{r}$ such that $\left\|\left[\rho_{k}, \rho\right]\right\|<\left\|\left[\rho_{r}, \rho\right]\right\|$ and there is no player $p_{z}, 1 \leq k, r, z \leq s,\|\{k, r, z\}\|=3$, with $\left\|\left[\rho_{z}, \rho\right]\right\|<\left\|\left[\rho_{r}, \rho\right]\right\|$. If there is more than one player fulfilling this condition for player $p_{k}$, and player $p_{j}$ is one of them, choose player $p_{k}$ other than $p_{j}$. Set $m_{r}:=\left(\rho_{k}-\rho_{r}\right) / 2$.

Step 4.3. Find any players $p_{k}$ and $p_{r}$ such that $\left\|\left[\rho_{k}, \rho^{\prime}\right]\right\|<\left\|\left[\rho_{r}, \rho^{\prime}\right]\right\|$ and there is no player $p_{z}, 1 \leq k, r, z \leq s,\|\{k, r, z\}\|=3$, with $\left\|\left[\rho_{z}, \rho^{\prime}\right]\right\|<\left\|\left[\rho_{r}, \rho^{\prime}\right]\right\|$. If there is more than one player fulfilling this condition for player $p_{k}$, and player $p_{j}$ is one of them, choose player $p_{k}$ other than $p_{j}$. Set $m_{r}:=\left(\rho_{k}-\rho_{r}\right) / 2$.

Step 5. Assign portion $C_{j}=\left[\lambda, \lambda_{j}+m_{\ell}\right]$ to player $p_{j}$.

Step 6. If $\rho=\rho^{\prime}$, assign portion $C_{k}=\left[\rho_{k}-m_{r}, \rho\right]$ to player $p_{k}$, else assign portion $C_{k}=\left[\rho_{k}-m_{r}, \rho^{\prime}\right]$ to player $p_{k}$.

Step 8. If $\rho=\rho^{\prime}$, set $C^{\prime}:=\left[\lambda_{j}+m_{\ell}, \rho_{k}-m_{r}\right]$ and $\rho:=\rho_{k}-m_{r}$, else set $C^{\prime}:=\left[\lambda_{j}+\right.$ $\left.m_{\ell}, \rho_{k}-m_{r}\right] \cup\left[\rho^{\prime}, \rho\right]$ and $\rho:=\rho-\rho^{\prime}+\rho_{k}-m_{r}$. Set $\lambda:=\lambda_{j}+m_{\ell}, \rho^{\prime}:=\rho$, and $s:=s-2$.

Step 9.2. Find any players $p_{j}$ and $p_{k}$ such that $\left\|\left[\rho_{j}, \rho\right]\right\|<\left\|\left[\rho_{k}, \rho\right]\right\|$ and there is no player $p_{\ell}, 1 \leq j, k, \ell \leq s,\|\{j, k, \ell\}\|=3$, with $\left\|\left[\rho_{\ell}, \rho\right]\right\|<\left\|\left[\rho_{k}, \rho\right]\right\|$. Set $m_{r}:=$ $\left(\rho_{j}-\rho_{k}\right) / 2$.

Step 9.3. Assign portion $C_{j}=\left[\rho_{j}-m_{r}, \rho\right]$ to player $p_{j}$. Let player $p_{j}$ drop out.

Step 9.4. $\quad$ Set $\rho:=\rho_{j}-m_{r}$ and $C^{\prime}:=[\lambda, \rho]$. Set $s:=s-1$.

Figure 4: Modified steps in the first iteration of the protocol in Figure 3 to achieve a strong fair division for $n>3$ players.

\section{Proof of Theorem 3.5}

In this section, we determine the degrees of guaranteed envy-freeness of the proportional cakecutting protocols listed in Table 1 Thus, we prove Theorem 3.5 via Lemmas 5.1 through 5.6. We investigate proportional protocols only, because proportional cake-cutting protocols have a DGEF of at least $n$ according to Proposition 3.3 and, thus, show some degree of fairness already.

\footnotetext{
${ }^{18}$ Recall that we assumed the axiom of positivity, which requires nonempty pieces of cake to have a nonzero value for each player, see Footnote 3 for more discussion of this point.
} 
Over the years, several cake-cutting protocols have been proven to be proportional and finite bounded for any number $n$ of players. A detailed description of various finite bounded proportional protocols can be found in the books by Brams and Taylor [BT96] and Robertson and Webb [RW98]. Our analysis of the protocols in Table 1 provides a basis for further algorithmic improvements in terms of the degree of guaranteed envy-freeness, and the protocol in Figure 3 is a first step in this direction.

In the following subsections, we give a brief description of the protocols listed in Table 1 and provide a detailed analysis of their DGEF. Note that the value of cake $C$ to be divided is normalized such that $v_{i}(C)=1$ for all players $p_{i}$ with $1 \leq i \leq n$.

\subsection{Last Diminisher}

The protocol works as follows: The first player cuts a piece he or she considers being worth exactly $1 / n$ in his or her measure. This piece is given to the $n-1$ other players, one after the other. Now, each player has the choice to either pass the piece on as it is, or to trim it before passing it on. If a player considers the piece to be worth more than $1 / n$, he or she trims it to exactly $1 / n$ according to his or her measure. When the last player has evaluated this piece, it is given to the player who was the last trimming it, or to the player who cut it in the first place if no trimmings have been made. The trimmings are reassembled with the remainder of the cake, and the procedure is applied in the same way for the $n-1$ remaining players and the reassembled remainder of the cake. This process is repeated until only two players remain. In the final round (of $n-1$ rounds in total), these last two players apply the simple cut-and-choose protocol to the remainder of the cake. For guaranteeing each player a proportional share of the cake, the order of the players is of no significance.

Lemma 5.1 The Last Diminisher protocol has a degree of guaranteed envy-freeness of $2+n(n-1) / 2$.

Proof. Concerning the analysis of the degree of guaranteed envy-freeness, it is quite evident that in the first round $n-1$ envy-free-relations are guaranteed, since each of the $n-1$ players not receiving the first piece consider this piece to be of value at most $1 / n$. Analogously, in the $k$ th round, $1<k<n, n-k$ additional envy-free-relations are guaranteed. The number of guaranteed envyfree-relations is consecutively decreasing by one per round, as the players who already received a piece are not involved in the evaluation process of subsequent rounds (see the proof of Lemma 3.4). This sums up to $\sum_{i=1}^{n-1} i=n(n-1) / 2$ guaranteed envy-free-relations. In addition, in the final round one more guaranteed envy-free-relation is created, as the simple cut-and-choose protocol guarantees that both of the players will not envy each other. Finally, note that the player receiving the first portion is not involved in the evaluations of any other portions, but since the Last Diminisher protocol is proportional, this player too cannot envy each of the other players according to the argument in the proof of Proposition 3.3. Thus, one more guaranteed envy-free-relation must be added. Consequently, the Last Diminisher protocol guarantees $2+n(n-1) / 2$ envy-free-relations.

\subsection{Lone Chooser}

The Lone Chooser protocol was first proposed by Fink [Fin64] (as cited in [Saa70, BT96]). It can be described as follows. For two players, the protocol is just the simple cut-and-choose protocol. For 
$n>2$ players, the protocol has $n-1$ rounds. The first round simply describes the cut-and-choose protocol executed by players $p_{1}$ and $p_{2}$, which results in two pieces, $c_{1}$ and $c_{2}$, with $C=c_{1} \cup c_{2}$. Assuming player $p_{1}$ received piece $c_{1}$ and player $p_{2}$ received piece $c_{2}$, in the second round player $p_{1}$ has to divide piece $c_{1}$ with player $p_{3}$, and player $p_{2}$ has to divide piece $c_{2}$ with player $p_{3}$. To this end, $p_{1}$ cuts $c_{1}$ into three pieces each of which he or she considers to be worth at least $1 / 6$, and so does $p_{2}$ with $c_{2}$. Player $p_{3}$ then chooses one of the pieces of player $p_{1}$ and one of the pieces of player $p_{2}$, both being most valuable according to $p_{3}$ 's measure. This guarantees each of the players $p_{1}, p_{2}$, and $p_{3}$ a portion of at least $1 / 3$ in their measures. Carrying on in this way, when the final round has been entered, each of the players $p_{1}, p_{2}, \ldots, p_{n-1}$ is in possession of a portion that he or she considers to be worth at least $1 /(n-1)$ in his or her measure. Let us refer to those $n-1$ players as the "cutters" of round $n-1$. Finally, each of the cutters $p_{1}, \ldots, p_{n-1}$ cuts his or her portion into $n$ pieces each of value $1 /\left(n^{2}-n\right)$, and player $p_{n}$, the "chooser" of round $n-1$, chooses one piece of highest value (according to his or her measure) from each plate of the $n-1$ cutters of this round.

Lemma 5.2 The Lone Chooser protocol has a degree of guaranteed envy-freeness of $n$.

Proof. In the course of the Lone Chooser protocol, none of the players evaluate the portion of any of the other players, which determines the DGEF of the Lone Chooser protocol to be $n$ as a result of Lemma 3.4.

\subsection{Lone Divider}

A complete algorithmic description of this protocol would be rather comprehensive. That is why we will give just a rough sketch of the procedure. For a more detailed description, the reader is referred to Kuhn [Kuh67], see also, e.g., [BT96, RW98, Daw01].

The Lone Divider protocol works as follows: Some player $p_{d}, 1 \leq d \leq n$, is chosen to be the firstround "divider." The divider cuts cake $C$ into $n$ portions that he or she considers each to be worth $1 / n$, i.e., $v_{d}\left(C_{j}\right)=1 / n$ with $1 \leq j \leq n$. Subsequently, all other players (the first-round "choosers") are asked to identify any of the $n$ portions they find acceptable, that is, each player identifies all portions that are worth at least $1 / n$ in this player's measure. Obviously, every chooser $p_{c}, c \neq d$, needs to accept at least one portion $C_{j}$. Depending on the players' choices, there are different ways for how the protocol continues. In the simplest case, the players' choices allow for a fully decidable division, i.e., for a division such that each chooser $p_{c}$ receives one of the portions he or she previously identified as being acceptable. Divider $p_{d}$ then receives the portion that has not been assigned to any of the choosers. All players drop out and the division is complete. However, if the players' choices do not allow a fully decidable division, there is either a partially decidable division (which means that only some — not all—of the choosers are assigned a portion and drop out), or a fully undecidable division (which means that there are at least two portions that have not been identified as being acceptable by any of the choosers and that none of the choosers receive a portion). In the case of a partially decidable division, the choosers accomplish only a partial allocation of the cake, i.e., those choosers that identified acceptable portions in a nonconflicting way are assigned a portion they have marked as being acceptable and drop out. In addition, this round's divider is assigned any one of the other portions and drops out, the remaining portions (that could 
not be assigned to players) are reassembled, and a new round with the remaining players, among which a new divider is to be chosen, is started. In the case of a fully undecidable division, none of the choosers are assigned a portion; only this round's divider receives one of the two portions that have not been identified as being acceptable by any of the choosers and drops out, and a new round with the remaining players, among which a new divider is to be chosen, is started in which the remaining cake is divided. This procedure is repeated until the whole cake has been allocated. Note that in each round at least this round's divider is assigned a portion and drops out.

It is easy to see that the Lone Divider protocol is finite bounded and proportional.

Lemma 5.3 The Lone Divider protocol has a degree of guaranteed envy-freeness of $2 n-2$.

Proof. The analysis of the degree of guaranteed envy-freeness is done by a worst-case scenario in terms of the number of existing envy-free-relations. We claim that the maximum number of guaranteed envy-free-relations exists in the case that every chooser $p_{c}$ marks $n-1$ portions as being acceptable in the first round. In the following, let us refer to this situation as the "worstcase scenario." In this scenario, the rules of the protocol imply that a proportional division will be achieved in the very first round (i.e., in this worst case scenario the first round results in a fully decidable division), and the following envy-free-relations are guaranteed to exist in this case. The divider will not envy any of the choosers, as he or she considers each of the portions to be $1 / n$, resulting in $n-1$ guaranteed envy-free-relations. Furthermore, none of the $n-1$ choosers will envy the player (be it the divider or any of the other choosers) that received the portion he or she considers to be not acceptable, leading to additional $n-1$ guaranteed envy-free-relations. Since the DGEF is the maximum number of envy-free-relations that are guaranteed to exist in every case, the DGEF of the Lone Divider protocol is at most $2 n-2$. To argue that the scenario given above indeed represents the worst case for $n \geq 3$ players (and so the DGEF is equal to $2 n-2$ ), we will consider all possible cases different from the worst-case scenario. We will show that in each of these cases the number of existing envy-free-relations is, in fact, higher than $2 n-2$. This implies that none of these other cases considered represent a worst-case scenario.

For notational convenience, we will use the term "case-enforced envy-free-relation" to refer to the number of those envy-free-relations that necessarily must exist in any of these cases, regardless of which particular valuation functions the players have (other than what was causing the respective case to occur). Recall that the term "guaranteed envy-free-relation" is reserved for the number of envy-free-relations that necessarily exist in the worst case (and-as we will see-none of the cases below will describe the worst case). Thus, the term "case-enforced envy-free-relation" is more general than and includes the term "guaranteed envy-free-relation": The number of caseenforced envy-free-relations in the worst case (i.e., the minimum number of case-enforced envyfree-relations, where the minimum is taken over all possible cases) is exactly the number of guaranteed envy-free-relations.

Case 1: The first round results in a fully decidable division. Consider the following two subcases.

Case 1.1: The total number of inacceptable portions is greater than in the worst-case scenario. Specifically, let us look at the situation that exactly one of the choosers considers just one more portion as being inacceptable than in the worst-case scenario. 
This change decrements the total number of acceptable portions by one and thus creates one additional case-enforced envy-free-relation, since this chooser will not envy the player receiving this particular portion. However, since the number of guaranteed envyfree-relations of the worst-case scenario persists also in this case, increasing the total number of inacceptable portions also increases the total number of case-enforced envyfree-relations. Thus, the present case does not describe a worst-case scenario.

Case 1.2: The total number of acceptable portions is greater than in the worst-case scenario. Specifically, let us look at the situation that exactly one of the choosers considers just one more portion as being acceptable than in the worst-case scenario. This chooser then accepts all portions, and thus necessarily considers each of the portions to be worth exactly $1 / n$. Accordingly, this chooser does not envy any of the other players, resulting in $n-2$ additional case-enforced envy-free-relations. In particular, since this chooser still does not envy the player he or she did not envy in the worst-case scenario, incrementing the total number of acceptable portions by just one increases the total number of caseenforced envy-free-relations by one (if $n=3$ ) or even more (if $n>3$ ). Thus, the present case does not describe a worst-case scenario.

Case 2: The first round does not result in a fully decidable division. Thus, the protocol runs over more than one round. For $n=3$ players, an additional round would be caused only by a fully undecidable division. For $n>3$ players, additional rounds are caused by either a fully undecidable division or a partially decidable division. In every round, at least the divider of this round is assigned a portion and drops out. On the part of the choosers, entering a new round creates additional case-enforced envy-free-relations the number of which depends on the present circumstances. Simply put, every additional round will increase the total number of case-enforced envy-free-relations compared with the worst-case minimum of $2 n-2$. This justifies why a division obtained by the execution of more than one round does not present a worst-case scenario.

To see why running more than one round will lead to more than $2 n-2$ case-enforced envyfree-relations, let us first, in Cases 2[1] and 222, have a closer look at the case-enforced envyfree-relations created in each nonfinal round if more than one round is executed in total. The final round will be handled separately in Case 23, In particular, if there are exactly two rounds, the total number of case-enforced envy-free-relations created is the sum of those explained in the first paragraph of either Case 211 or Case 2/2 (which describe the number of case-enforced envy-free-relations created in the first round) and those explained in Case 23 (which describes the number of case-enforced envy-free-relations created in the final round).

Case 2.1: In the case of a fully undecidable division of the cake as the result of the first round, the first-round divider receives one of the portions that have not been marked as being acceptable by any of the choosers and drops out. All $n-1$ choosers enter the second round and will not envy the divider of the first round, resulting in $n-1$ case-enforced envy-free-relations. Moreover, the first-round divider will not envy at least one of the $n-1$ other players, since there must be at least one player he or she does not envy, which 
follows from the proof of Proposition 3.3 Thus, $n$ case-enforced envy-free-relations result from the first round in this case.

Note that Proposition 3.3 can be applied only to the first-round divider. Thus, analogously to the above argument, for every additional round with $s<n$ players that is caused by a fully undecidable division and that is not the final round, $s-1$ case-enforced envy-free-relations need to be added. An analysis of the final round follows in Case 233.

Case 2.2: In the case of a partially decidable division of the cake as the result of the first round, let $K$ denote the set of those $k$ players, $1<k<n-1$, that are in conflict with each other concerning the portions they identified as being acceptable in this first round. Let $L$ be the set of the $\ell$ remaining players, where $1<\ell<n-1$ and $n=k+\ell$. The players in $L$ can divide a part of the cake without any conflict, i.e., each of the players in $L$ is assigned one of the portions he or she identified as being acceptable. Note that $\ell=1$ would represent Case 211, and that the divider always is one of the players in $L$. Following the protocol, each of the players in $L$ receives a portion and drops out while each of the players in $K$ enters the second round. Since none of the players in $K$ would accept any of the portions the players in $L$ have received, each of the players in $K$ does not envy any of the players in $L$, resulting in $k \ell \geq k+\ell=n$ case-enforced envy-freerelations. The players in $L$ (except for the first-round divider) that are assigned a portion and drop out in the first round are each guaranteed one envy-free-relation due to the argument in the proof of Proposition 3.3, summing up to $\ell-1$ additional case-enforced envy-free-relations. Moreover, the first-round divider will not envy any of the $\ell-1$ other players in $L$, resulting in $\ell-1$ more case-enforced envy-free-relations. Consequently, $2(\ell-1)+k \ell$ case-enforced envy-free-relations result from the first round in this case.

Now consider any additional round with $s=k^{\prime}+\ell^{\prime}$ players that is caused by a partially decidable division of the cake and that is not the final round, where $k^{\prime}$ players are in conflict with each other and $\ell^{\prime}$ players accomplish a partial allocation of the cake. Analogously to the above argument (except that Proposition 3.3 is no longer applicable), $k^{\prime} \ell^{\prime}$ case-enforced envy-free-relations are to be added for the $k^{\prime}$ players being in conflict, and $\ell^{\prime}-1$ case-enforced envy-free-relations are to be added for this round's divider. An analysis of the final round follows in Case 23

Case 2.3: Aside from the case-enforced envy-free-relations that are created by executing a nonfinal round, additional case-enforced envy-free-relations are created in the final round, in which even the last player receives a portion. In each case, the final round is characterized by providing a fully decidable division. Consequently, considering a final round with $s$ players at least $2(s-1)$ case-enforced envy-free-relations are created according to Cases 111 and 12

As a result, in the example of the protocol running a second round, either $n+2(s-1)=n+$ $2(n-2)$ with $s=n-1$ (see Case 21), or $2(\ell-1)+k \ell+2(s-1)=k \ell+2(n-2) \geq n+2(n-2)$ with $s=k$ (see Case 22) envy-free-relations are case-enforced. Thus, since $n \geq 3$, a second round yields at least $n+2(n-2)>2 n-2$ case-enforced envy-free-relations in total. As indicated above, every additional round increases the number of case-enforced envy-free-relations even more. 
Cases 1 and 2 (and their subcases) completely characterize all situations different from the worst case scenario, since their number of case-enforced envy-free-relations is always greater than $2 n-2$, the number of envy-free-relations guaranteed to exist in the scenario given in the first paragraph of this proof. This justifies that this scenario indeed represents the worst case. Note that the protocol does not require any of the choosers to value any of the portions they marked as being acceptable (i.e., the only information provided on these portions is that they are considered to be worth at least $1 / n$ ), and thus, according to the proof of Lemma 3.4, the DGEF of the Lone Divider protocol is $2 n-2$.

\subsection{Cut Your Own Piece}

The protocol works as follows: Every player $p_{i}$ marks $n$ adjacent pieces each valued $1 / n$ in his or her measure, resulting in $n(n-1)$ marks in total. Afterwards, a cut is made at between $n-1$ and $2(n-1)$ of the existing marks, resulting in at least $n$ adjacent pieces. Each player then is assigned a portion that contains at least one of the pieces he or she marked beforehand plus some optional supplement. There are different strategies for how to make cuts such that the resulting division is fair. The number of cuts to be made depends on the strategy chosen. Note that there always is at least one strategy that guarantees each player $p_{i}$ a portion valued at least $1 / n$ according to his or her measure, i.e., $v_{i}\left(C_{i}\right) \geq 1 / n$ for all $1 \leq i \leq n$ [Ste69]. If $n-1$ cuts are made (at $n-1$ of the existing marks), each player's portion consists of exactly one piece which is worth at least $1 / n$ according to his or her measure.

Lemma 5.4 The Cut Your Own Piece protocol has a degree of guaranteed envy-freeness of $n$ if no strategy is specified, and a degree of guaranteed envy-freeness of $2 n-2$ for the "left-right strategy" (which, for convenience, will be explained in the proof). Moreover, for this protocol no strategy can give a better DGEF than $2 n-2$.

Proof. As mentioned above, there are different strategies for how to make cuts such that the resulting division is fair. If no particular strategy is given, this protocol has a DGEF of only $n$ (according to Lemma 3.4), since all players made their marks independently, and none of the players were asked to give an evaluation of the marks of any of the other players. Hence, only the minimum of $n$ envy-free-relations can be guaranteed. Steinhaus [Ste69] did not mention a strategy for how to achieve a simple fair division; he just mentioned that there always exists at least one.

However, when we consider a strategy that always assigns the leftmost (with respect to the interval $[0,1]$ ) smallest piece to the player that marked this piece as being of value $1 / n$, and the rightmost smallest piece to the player that marked this piece as being of value $1 / n$, this protocol guarantees at least $2 n-2$ envy-free-relations. We call this strategy the left-right strategy.

In more detail, applying the left-right strategy we assign the leftmost piece to the player that marked the smallest piece starting at 0 , and we assign the rightmost piece to the player that marked the smallest piece finishing at 1 . That way it is guaranteed that the $n-2$ remaining players each consider the part of the cake between the assigned leftmost piece and the assigned rightmost piece as being worth at least $(n-2) / n$. Thus, this subpart of the cake can be allocated to these $n-2$ players according to the marks made in the first instance. Note that if the player that marked the leftmost 
smallest piece happens to be the same as the one that marked the rightmost smallest piece, the rightmost piece is given to the player that marked the second smallest piece finishing at 1 . If several marks for the leftmost (respectively, for the rightmost) smallest piece coincide, any mark can be chosen, without loss of generality. In this example, the terms "piece" and "portion" can be used interchangeably, as this protocol assigns contiguous portions.

When applying the left-right strategy as described above, it is guaranteed that the player receiving the leftmost smallest piece is not envied by any of the $n-1$ remaining players, since all of them value this piece at most $1 / n$ according to their measures. Analogously, it is guaranteed that the $n-2$ players in the "middle" do not envy the player receiving the rightmost smallest piece as they value this piece at most $1 / n$. Note that if the player that marked the leftmost smallest piece is the very same as the one that marked the rightmost smallest piece, this player may envy the player receiving the rightmost piece, since in this case the rightmost piece to be assigned is just the second smallest piece finishing at 1 . Thus, only $n-2$ envy-free-relations can be guaranteed with respect to the player receiving the rightmost piece. However, one more guaranteed envy-free-relation needs to be added, as the player that marked and is assigned the leftmost smallest piece cannot envy each of the other players (according to the argument in the proof of Proposition 3.3). Consequently, $2 n-2$ envy-free-relations can be guaranteed in total.

The DGEF achieved for the Cut Your Own Piece protocol by the application of the left-right strategy cannot be enhanced by any other strategy. The latter is due to the fact that all pieces have been marked without any mutual evaluations (as already mentioned above), and that no common boundaries other than the left border of the leftmost piece and the right border of the rightmost piece (with respect to the interval $[0,1]$ ), which could be used for subsequent comparisons of the guaranteed sizes and thus values of the pieces marked, are known.

\subsection{Divide and Conquer}

The Divide and Conquer protocol, which was first presented by Even and Paz [EP84] (see also, e.g., [RW98]), is based on the idea of dividing cake $C$ by simultaneously partitioning disjoint parts of $C$. The procedure slightly differs depending on whether the number of players is even or odd.

If there is an even number of players, say $n=2 k$ for some integer $k$, all players but one divide cake $C$ in the ratio $k / k$ by a single cut, yielding two pieces of equal value for each of these $n-1$ players. The noncutter identifies either the piece to the left of the middle cut (with respect to the interval $[0,1]$ ), or the piece to the right of the middle cut as being worth at least half of the cake according to his or her measure, and then continues dividing this piece with those $k-1$ cutters whose cuts fall within this piece. The other piece will be divided among the $k$ remaining cutters. That is, a new round is started in which those two pieces of $C$ are divided among $k$ players each, simultaneously but independently of each other.

If there is an odd number of players, say $n=2 k+1$, all players but one divide cake $C$ in the ratio $k /(k+1)$ by a single cut. The noncutter identifies either the piece to the left of the $k$ th cut as being worth at least $k /(2 k+1)$, or the piece to the right of the $k$ th cut as being worth at least $(k+1) /(2 k+1)$. Accordingly, the noncutter continues dividing either the piece to the left of the $k$ th cut with those $k-1$ cutters whose cuts fall within this piece, or the noncutter divides the piece to the right of the 
$k$ th cut with those $k$ cutters whose cuts fall within this piece. In both cases, the other piece will be divided among all the remaining cutters.

In this way, the procedure is applied recursively until just one player remains in each subprocedure, i.e., until all the cake has been allocated to the players. Note that in the case of $n=2$, this is just the simple cut-and-choose protocol.

Brams, Jones, and Klamler [BJK07] present a finite bounded proportional cake-cutting protocol that is based on a divide-and-conquer strategy, and focuses on minimizing the number of players the most-envious player may envy. The major difference to the protocol described above lies in the way of splitting the piece of a particular subprocedure into two subpieces. While the original Divide and Conquer protocol suggests to use one of the cuts made by the cutters, the Minimal-Envy Divide and Conquer protocol suggests to conduct one more cut strictly between the cut chosen by the Divide and Conquer protocol and the very next right neighboring cut (according to the interval $[0,1]$ ), and then to use this additional cut for splitting the particular piece of the cake into two subpieces for the following round if there is any (or to be assigned if this has been the final round).

Lemma 5.5 The Divide and Conquer protocol and the Minimal-Envy Divide and Conquer protocol both have a degree of guaranteed envy-freeness of $n \cdot\lfloor\log n\rfloor+2 n-2^{\lfloor\log n\rfloor+1}$.

Proof. The Divide and Conquer protocol is recursively defined. Put simply, in each subprocedure the given subpart of the cake is divided into two pieces and so are the players into two groups, the procedure then is applied recursively again and again to the resulting pieces and related players until in each subprocedure just one player remains. In each round, every player participating in any of the subprocedures of this round will not envy at least one of the players continuing with the corresponding other piece, as this other piece is of no more value (according to his or her measure) than the one he or she is continuing with. Thus, in each round, for each player involved in this round, one envy-free-relation is guaranteed to exist. Note that, for each subprocedure in any round except the final one, the numbers of players to be continued with in the resulting two subprocedures of the following round depend on whether the total number of players involved in the given subprocedure is even or odd.

From these remarks it follows that the Divide and Conquer protocol's degree of guaranteed envy-freeness, call it $d(n)$ for $n$ players, can be described by the following recurrence:

$$
\begin{array}{lll}
d(1) & =0, & \\
d(n)=d(k)+d(k)+2 k & & \text { for } n=2 k, \\
d(n)=d(k)+d(k+1)+2 k+1 & & \text { for } n=2 k+1 .
\end{array}
$$

Apparently, this recurrence relation can be simplified to:

$$
\begin{aligned}
& d(1)=0, \\
& d(n)=d(\lfloor n / 2\rfloor)+d(\lceil n / 2\rceil)+n \quad \text { for } n \geq 2 .
\end{aligned}
$$

The recurrence in (5.a) and similar versions are well known to occur also in other contexts 19 It

\footnotetext{
${ }^{19}$ For example, they also occur in the context of evaluating the number of comparisons made by various sorting algorithms that are based on a divide-and-conquer strategy. In particular, this recurrence expresses the number of comparisons done by the standard merge-sort algorithm.
} 
is a matter of routine (see, e.g., [GKP94]) to solve it (i.e., to bring it into closed form):

$$
d(n)=n \cdot\lfloor\log n\rfloor+2 n-2^{\lfloor\log n\rfloor+1} \text { for } n \geq 1 .
$$

How does Equation (5.b) reflect the guaranteed number of envy-free-relations of the Divide and Conquer protocol? As mentioned before, this protocol can be considered as a collection of several subprocedures that altogether yield a proportional division of the given cake. This collection of subprocedures can be represented as a balanced binary tree (called the "recursion tree"), because in every subprocedure the given subpart of the cake is cut into two pieces for which the procedure is applied recursively again and again until just one player remains in each resulting subprocedure. Since the depth of a balanced binary tree is logarithmic in the number of leaves, $\lceil\log n\rceil$ rounds are performed in total. If the number $n$ of players is not a power of two, every round except for the last one (i.e., $\lfloor\log n\rfloor$ rounds) is represented by a completely filled level of the binary tree in terms of the number of players, since all $n$ players are participating in these rounds-in different subprocedures though. Note that $n$ is a power of two if and only if it holds that $\lceil\log n\rceil=\lfloor\log n\rfloor$ (i.e., the final round is numbered $\lfloor\log n\rfloor$ ), and only in this case, all $n$ players are involved in each of the rounds, even in the final round.

Recall that, in each round, every participating player will not envy at least one of the players continuing with the particular other piece, since he or she considers this piece to be of no more value than the one he or she is continuing with, i.e., in each round one guaranteed envy-free-relation is created on behalf of each of the participating players. For this reason, $n$ envy-free-relations are guaranteed to be created in each of the first $\lfloor\log n\rfloor$ rounds. This is because in subsequent rounds the particular subparts to be divided will never get bigger again, and once two players have ended up in different subprocedures, they will never meet again in the same subprocedure of any of the following rounds. Moreover, once a player has ended up in some group, he or she will not make future evaluations of pieces of the cake to be divided among the players in the other group. Thus, those envy-free-relations that result from any of the first $\lfloor\log n\rfloor$ rounds are guaranteed to persist until all the cake has been allocated. However, it cannot be determined which of the players continuing with the other piece of the particular subpart is not envied, since no evaluations of the pieces created in other subprocedures are made. In accordance with the proof of Lemma 3.4 the latter also justifies why no more envy-free-relations can be guaranteed. Hence, it can just be guaranteed that each player does not envy at least one of the players continuing with the other piece. Summing up, as exactly $n$ guaranteed envy-free-relations are created in each of the first $\lfloor\log n\rfloor$ rounds, $n \cdot\lfloor\log n\rfloor$ guaranteed envy-free-relations are created over all rounds, except for the final round if $n$ is not a power of two. Note that if $n$ is a power of two, the $(\log n)$ th round is the final round and Equation (5.b) simplifies to $d(n)=n \cdot \log n$, so in this case we are done.

In contrast, if $n$ is not a power of two then less than $n$ players will be involved in the final round (i.e., in the round numbered $\lceil\log n\rceil=\lfloor\log n\rfloor+1$ ), since in that case there is at least one subprocedure that involves an odd number of players. More specifically, in this case the number of players involved in the final round can be expressed by the term $2 n-2^{\lfloor\log n\rfloor+1}$, where $2^{\lfloor\log n\rfloor+1}$ specifies the number of players that would be involved in the final round if the binary recursion tree would be a full binary tree, i.e., if all $n$ players would be involved in the final round.

In order to analyze the final round for $n$ not being a power of two in detail, let $i$-subprocedure denote a subprocedure involving exactly $i$ players. A 3-subprocedure can occur only in the second- 
to-last round, and if it occurs then one of its three players cannot be participating in the final round, i.e., only two out of three players are proceeding to the final round. Since in a balanced binary tree the depth of all leaves differs by at most one, the second-to-last round can have only either 2-subprocedures and/or 3-subprocedures, or 4-subprocedures and/or 3-subprocedures. Regarding a second-to-last round with at least one 3-subprocedure and any number of 2-subprocedures (which can happen only if $n$ is not a power of two), the number of players involved in the final round will be twice the number of 3-subprocedures occurring in the second-to-last round. Regarding a second-to-last round with at least one 3-subprocedure and any number of 4-subprocedures (which again can happen only if $n$ is not a power of two), the number of players involved in the final round will be twice the number of 3-subprocedures plus four times the number of 4-subprocedures. Consequently, the number of 3-subprocedures and the number of 4-subprocedures in the second-tolast round determine how many players are participating in the final round, and thus also determine the number of guaranteed envy-free-relations to be created in the final round.

If $n$ is not a power of two, analogously to the argument for the first $\lfloor\log n\rfloor$ rounds, also in the final round one guaranteed envy-free-relation is created with respect to each participating player. Thus, for any number $n \geq 1$ of players, at least $2 n-2^{\lfloor\log n\rfloor+1}$ guaranteed envy-free-relations are created in the $(\lfloor\log n\rfloor+1)$ th round 20 According to the proof of Lemma 3.4 no more than that many envy-free-relations can be guaranteed. Altogether, this sums up to $n \cdot\lfloor\log n\rfloor+2 n-2^{\lfloor\log n\rfloor+1}$ guaranteed envy-free-relations in total, which is the number stated in Equation (5.b).

The degree of guaranteed envy-freeness of the Minimal-Envy Divide and Conquer protocol can be shown just as for the original Divide and Conquer protocol. The difference in the way of splitting the particular piece of the cake into two subpieces does not affect the number of guaranteed envy-free-relations. Consequently, although the Minimal-Envy Divide and Conquer protocol does decrease envy according to the definition of Brams, Jones, and Klamler [BJK07] (see Section 6 for more discussion of this point), its DGEF is $n \cdot\lfloor\log n\rfloor+2 n-2^{\lfloor\log n\rfloor+1}$, just as for the original Divide and Conquer protocol.

\subsection{Recursive Divide and Choose}

This protocol has been presented by Tasnádi [Tas03] and describes a recursive procedure for how to always achieve a proportional division. It works as follows: In the case of $n=2$, this is just the simple cut-and-choose protocol. In the case of $n=3$, one of the players, the "divider," divides the cake into three equal pieces according to his or her measure, and each of the two other players, the "choosers," marks two pieces he or she considers to be worth the most, where ties may be broken arbitrarily. If both choosers marked the same two pieces, they divide these by applying the simple cut-and-choose protocol, and the divider receives the remaining piece. If the choosers marked different pieces, they divide the piece they both have marked via the simple cut-and-choose protocol, and each of the choosers divides the piece marked by just him- or herself with the divider, again via applying the simple cut-and-choose protocol.

In the case of $n>3$ players, this procedure is repeated recursively until all comes down to the simple cut-and-choose protocol involving two players only. In more detail, in the first round the

\footnotetext{
${ }^{20}$ Note that $n$ is a power of two if and only if $2 n-2^{\lfloor\log n\rfloor+1}=0$, and that in this case there is no $(\lfloor\log n\rfloor+1)$ th round.
} 
divider cuts the cake into $n$ equal pieces according to his or her measure, and each of the $n-1$ choosers marks $n-1$ pieces he or she considers to be worth the most, where ties may be broken arbitrarily. Afterwards, a new round is started and each of the $n$ pieces is divided among those $n-1$ choosers that identified this piece as being acceptable. Concerning pieces that have been marked by less than $n-1$ choosers, the divider fills out these empty slots by an appropriate number of clones. In other words, each of the $n$ pieces enters the next round of the protocol and induces a new subprocedure in the scope of which the particular piece is being divided among $n-1$ players. All $n$ subprocedures are executed simultaneously but independently of each other. Note that if in the very first round all $n-1$ choosers marked the same $n-1$ pieces as being acceptable than there is exactly one piece that has not been marked by any of the choosers. In this case, this piece is directly assigned to the divider and the divider drops out, whereas all choosers enter the next round for dividing the $n-1$ remaining pieces among them.

Analogously, in the $k$ th round, $1<k<n$, there are $\prod_{i=2}^{k}(n-i+2)$ subprocedures, i.e., $\prod_{i=2}^{k}(n-i+2)$ pieces are to be divided simultaneously but independently among $n-k+1$ players each. In every subprocedure any one player is determined to be the divider and cuts the particular piece of this subprocedure into $n-k+1$ equal subpieces according to his or her measure. Afterwards, each of the $n-k$ choosers of this particular subprocedure marks $n-k$ pieces he or she considers to be worth the most, where ties may be broken arbitrarily. Each of the $n-k+1$ pieces will induce a new subprocedure in the next round and will be divided among those $n-k$ players that marked this piece as being acceptable — where the divider fills out all empty slots regarding pieces that have been marked by less than $n-k$ choosers. Again, if in some round $k, 1<k<n$, in any of the subprocedures, all $n-k$ choosers agree on the same $n-k$ pieces, then there will be exactly one piece that has not been marked by any of the choosers. In this case, the unmarked piece is directly assigned to the divider of this particular subprocedure, and in the following rounds, the divider will not be involved in any of the subprocedures that results from this one, i.e., only $n-k$ pieces enter the next round, in which these are to be divided among the $n-k$ choosers of the previous round. Nevertheless, this divider will enter the next round and will participate in all those subprocedures that result from procedures he has not dropped out from yet.

Applying this procedure recursively until only two players remain in each subprocedure (i.e., running $n-1$ rounds), and dividing the corresponding piece of the cake between these two via the simple cut-and-choose protocol, the Recursive Divide and Choose protocol provides a proportional division of the cake.

Lemma 5.6 The Recursive Divide and Choose protocol has a degree of guaranteed envy-freeness of $n$.

Proof. For $n \geq 3$ players, no evaluations of entire portions are made-except for the one special case when the first-round divider drops out in the very first round (which, for the sake of self-containment, will be considered separately below) - and thus the scenario from the proof of Lemma 3.4 is applicable. Simply put, only $n$ envy-free-relations can be guaranteed in every case due to the argument in the proof of Proposition 3.3. as the missing evaluations of entire portions allow for any valuation functions, for example, those described in the proof of Lemma 3.4. If in the very first round all $n-1$ choosers agree on the very same $n-1$ pieces then the first-round divider 
will drop out with a portion that he or she values exactly $1 / n$ and that all other players value at most $1 / n$. This results in $n$ guaranteed envy-free-relations, since none of the $n-1$ choosers will envy the first-round divider and the first-round divider will not envy at least one of the other players by the argument in the proof of Proposition 3.3. However, in this case no more envy-free-relations can be guaranteed in the following rounds due to the argument given above. Consequently, the DGEF of the Recursive Divide and Choose protocol is $n$.

\section{Related Work and Discussion}

The analysis of envy-relations dates back at least to Feldman and Kirman [FK74]. In contrast to our approach, they consider the number of envy-pairs in already existing divisions with the intention of maximizing fairness afterwards via trading. In particular, they do not consider the design of cake-cutting protocols that maximize fairness. In the majority of cases, research in the area of cakecutting from an economic perspective is concerned more with the existence of certain divisions and their properties than with how to achieve these divisions.

A different approach measures the intensity of envy in terms of the distance between envied portions [Cha86].

More recently, Brams, Jones, and Klamler [BJK07] proposed to minimize envy in terms of the maximum number of players that a player may envy. Their notion of measuring envy differs from our notion of DGEF in various ways, the most fundamental of which is that their notion takes an "egalitarian" approach to reducing the number of envy-relations (namely, via minimizing the mostenvious player's envy, in terms of decreasing the number of this single player's envy-relations). In contrast, the DGEF aims at a "utilitarian" approach (namely, via minimizing overall envy, in terms of increasing the total number of guaranteed envy-free-relations among all players). That is to say that, although these notions may seem to be very similar at first glance, the approach presented in [BJK07] is not sensitive to a reduction in the number of envy-relations on the part of any other than the most-envious player, whereas the DGEF does take each single improvement into account and adapts accordingly. The DGEF, thus, is a more specific, more fine-tuned measure. Note also that Brams, Jones, and Klamler [BJK07] focus primarily on presenting a new protocol and less so on introducing a new notion for measuring envy.

Another approach is due to Chevaleyre et al. [CEEM07], who define various metrics for the evaluation of envy in order to classify "the degree of envy in a society," and they use the term "degree of envy" in the quite different setting of multiagent allocation of indivisible resources.

Besides, we stress that our approach of approximating envy-freeness differs from other lines of research that also deal with approximating fairness. For example, Lipton et al. [LMMS04] propose to seek for minimum-envy allocations of indivisible goods in terms of the value difference of the utility functions of envied players, and Edmonds and Pruhs [EP06b, EP06a] approximate fairness in cake-cutting protocols by allowing merely approximately fair pieces (in terms of their value to the players) and by using only approximate cut queries (in terms of exactness).

It may be tempting to seek to decrease envy (and thus to increase the DGEF) via trading, aiming to get rid of potential circular envy-relations. Although we do not consider trading to be an integral part of a cake-cutting protocol, let us for a moment digress to briefly discuss how trading may 
potentially affect the number of guaranteed envy-free-relations 21 Indeed, if the DGEF is lower than $n(n-1) / 2$, the number of guaranteed envy-free-relations can be improved to this lower bound, or to an even higher number, by resolving circular envy-relations (of which two-way envy-relations are a special case) by means of circular trades after the execution of the protocol. Thus, in this case, involving subsequent trading actions adds on the number of guaranteed envy-free-relations. Furthermore, having exactly $n(n-1) / 2$ guaranteed envy-free-relations after all circular envy-relations have been resolved, three more guaranteed envy-free-relations can be gained by applying an envyfree protocol (e.g., the Selfridge-Conway protocol) to the three most envied players, which yields to an overall lower bound of $3+n(n-1) / 2$ guaranteed envy-free-relations. To give an example for an even higher impact of trading, when circular trades indeed are involved after executing either the Divide and Conquer protocol or the Minimal-Envy Divide and Conquer protocol, their numbers of guaranteed envy-free relations can be improved to $(n(n+1) / 2)-1$, which follows from [BJK07].

On the other hand, if the DGEF of a proportional cake-cutting protocol is $n(n-1) / 2$ or higher (such as the DGEF of the protocol presented in Figure 3) then-depending on the protocol-circular envyrelations may not be guaranteed to exist, and if such cycles are not guaranteed to exist, trading has no impact on the number of guaranteed envy-free-relations.

However, as mentioned above, we consider trading not to be part of a cake-cutting protocol, though it might be useful in certain cases (for example, Brams and Taylor [BT96, page 44] mention that trading might be used "to obtain better allocations; however, this is not a procedure but an informal adjustment mechanism"). In particular, the notion of DGEF refers to (proportional) cakecutting protocols without additional trading, i.e., the DGEF is defined to make a statement on the performance of a particular protocol and not about all sorts of actions to be undertaken afterwards.

Although the well-known protocols listed in Table 1 have not been developed with a focus on maximizing the DGEF 22 linking their degrees of guaranteed envy-freeness to the lower bound provided by involving, e.g., the Selfridge-Conway protocol and guaranteed trading opportunities indicates that the development of cake-cutting protocols with a considerably higher DGEF or even with a DGEF close to the maximum of $n(n-1)$ poses a true challenge. That is why we feel that the enhanced DGEF of the protocol presented in Figure 3 constitutes a significant improvement.

\section{Conclusions}

Although different disciplines are engaged in the development of fair cake-cutting protocols for decades now, finite bounded protocols that guarantee an envy-free division for $n>3$ players are still a mystery. However, finite bounded protocols are the ones we are looking for in terms of practical implementations. That is why we in this paper have proposed to weaken the requirement

\footnotetext{
${ }^{21}$ To be specific here, all occurrences of "guaranteed envy-free-relations" in this and the next paragraph refer to those envy-free-relations that are guaranteed to exist after executing some cake-cutting protocol and in addition, subsequently, performing trades that are guaranteed to be feasible. This is in contrast with what we mean by this term anywhere else in the paper; "guaranteed envy-free-relations" usually refers to those envy-free-relations that are guaranteed to exist after executing the protocol only.

${ }^{22}$ Quite remarkably, without any trading actions and without involving, e.g., the Selfridge-Conway protocol the Last Diminisher protocol achieves with its DGEF almost (being off only by one) the trading- and Selfridge-Conway-related bound of $3+n(n-1) / 2$ mentioned above.
} 
of envy-freeness (as much as needed and as little as possible), while insisting on finite boundedness. To this end, we introduced the notion of degree of guaranteed envy-freeness for proportional cakecutting protocols. Based on this definition, we gave a survey of the DGEF in existing finite bounded proportional cake-cutting protocols, which shows that when one is trying to approximate the ideal of envy-freeness via the DGEF, there is quite a bit room for improvements. In particular, we expect that the concept of DGEF is suitable to extend the scope for the development of new finite bounded cake-cutting protocols by allowing to approximate envy-freeness step by step. In this context, we proposed a new finite bounded proportional cake-cutting protocol, explicitly demonstrated for $n=4$ and for arbitrary $n \geq 3$, which provides a significantly enhanced degree of guaranteed envy-freeness, compared with the status quo given by the survey in Table 1 (see also Section 5). In particular, our protocol has $\lceil n / 2\rceil-1$ more guaranteed envy-free-relations than the Last Diminisher protocol, which previously was the best finite bounded proportional cake-cutting protocol with respect to the DGEF. To achieve this significantly enhanced DGEF, our protocol makes use of parallelization with respect to the leftmost and the rightmost pieces. In this regard, adjusting the values of the pieces to be marked from $1 / n$ to $1 / s$ (with $s$ players still in the game) and applying an appropriate innerloop procedure is crucial to make the parallelization work. In addition to an enhanced DGEF, our protocol still has the other useful properties the Last Diminisher protocol is known to possess, such as strategy-proofness.

In general, we suggest to target improvements on the "level of envy-freeness" already in the design of cake-cutting protocols rather than trying to improve on the number of envy-free-relations afterwards for the division obtained (e.g., via trading [FK74, Cha86]). In terms of future research, this approach encourages to develop new protocols with even higher degrees of guaranteed envyfreeness - one may even think of modifications that focus on the development of protocols with "balanced envy-freeness" while keeping the DGEF high.

\section{Acknowledgments}

We are grateful to Ariel Procaccia for many interesting discussions and pointers to the literature. In particular, the second author thanks him for drawing his attention to the fascinating area of cakecutting during a visit to Hebrew University of Jerusalem, and he thanks Jeff Rosenschein for hosting this visit. We also thank Magnus Roos for helpful comments.

\section{References}

[Aki95] E. Akin. Vilfredo Pareto cuts the cake. Journal of Mathematical Economics, 24(1):23-44, 1995.

[BB04] J. Barbanel and S. Brams. Cake division with minimal cuts: Envy-free procedures for three persons, four persons, and beyond. Mathematical Social Sciences, 48(3):251-269, 2004.

[BJK07] S. Brams, M. Jones, and C. Klamler. Divide-and-Conquer: A proportional, minimal-envy cake-cutting procedure. In S. Brams, K. Pruhs, and G. Woeginger, editors, Dagstuhl Seminar 07261: "Fair Division". Dagstuhl Seminar Proceedings, November 2007. Available on-line at http://drops.dagstuhl.de/opus/volltexte/2007/1221/pdf/07261.BramsSteven.Pape:.1221.pdf.

[BT95] S. Brams and A. Taylor. An envy-free cake division protocol. The American Mathematical Monthly, 102(1):9-18, 1995. 
[BT96] S. Brams and A. Taylor. Fair Division: From Cake-Cutting to Dispute Resolution. Cambridge University Press, 1996.

[BTZ97] S. Brams, A. Taylor, and W. Zwicker. A moving-knife solution to the four-person envy-free cake-division problem. Proceedings of the American Mathematical Society, 125(2):547-554, 1997.

[CEEM07] Y. Chevaleyre, U. Endriss, S. Estivie, and N. Maudet. Reaching envy-free states in distributed negotiation settings. In Proceedings of the 20th International Joint Conference on Artificial Intelligence, pages 1239-1244. IJCAI, 2007.

[Cha85] A. Chauduri. Formal properties of interpersonal envy. Theory and Decision, 18:301-312, 1985.

[Cha86] A. Chauduri. Some implications of an intensity measure of envy. Social Choice and Welfare, 3(4):255-270, 1986.

[Daw01] C. Dawson. An algorithmic version of Kuhn's lone-divider method of fair division. Missouri Journal of Mathematical Sciences, 13(3):172-177, 2001.

[EP84] S. Even and A. Paz. A note on cake cutting. Discrete Applied Mathematics, 7:285-296, 1984.

[EP06a] J. Edmonds and K. Pruhs. Balanced allocations of cake. In Proceedings of the 47th IEEE Symposium on Foundations of Computer Science, pages 623-634. IEEE Computer Society, 2006.

[EP06b] J. Edmonds and K. Pruhs. Cake cutting really is not a piece of cake. In Proceedings of the 17th Annual ACM-SIAM Symposium on Discrete Algorithms, pages 271-278. ACM Press, 2006.

[Fin64] A. Fink. A note on the fair division problem. Mathematics Magazine, 37(5):341-342, 1964.

[FK74] A. Feldman and A. Kirman. Fairness and Envy. The American Economic Review, 64(6):9951005, 1974.

[Fol67] D. Foley. Resource allocation and the public sector. Yale Economic Essays, 7(1):45-98, 1967.

[FW79] A. Feldman and D. Weiman. Envy, wealth, and class hierarchies. Journal of Public Economics, 11(1):81-91, 1979.

[GKP94] R. Graham, D. Knuth, and O. Patashnik. Concrete Mathematics: A Foundation for Computer Science. Addison-Wesley, 2nd edition, 1994.

[Kuh67] H. Kuhn. On games of fair division. In M. Shubik, editor, Essays in Mathematical Economics in Honor of Oskar Morgenstern. Princeton University Press, 1967.

[LMMS04] R. Lipton, E. Markakis, E. Mossel, and A. Saberi. On approximately fair allocations of indivisible goods. In Proceedings of the 5th ACM conference on Electronic Commerce, pages 125-131. ACM Press, 2004.

[MIBK03] M. Magdon-Ismail, C. Busch, and M. Krishnamoorthy. Cake-cutting is not a piece of cake. In Proceedings of the 20th Annual Symposium on Theoretical Aspects of Computer Science, pages 596-607. Springer-Verlag Lecture Notes in Computer Science \#2607, 2003.

[Pro09] A. Procaccia. Thou shalt covet thy neighbor's cake. In Proceedings of the 21st International Joint Conference on Artificial Intelligence, pages 239-244. IJCAI, July 2009.

[RW97] J. Robertson and W. Webb. Near exact and envy free cake division. Ars Combinatoria, 45:97108, 1997. 
[RW98] J. Robertson and W. Webb. Cake-Cutting Algorithms: Be Fair If You Can. A K Peters, 1998.

[Saa70] T. Saaty. Optimization in Integers and Related Extremal Problems. McGraw-Hill, New York, 1970.

[Ste48] H. Steinhaus. The problem of fair division. Econometrica, 16:101-104, 1948.

[Ste49] H. Steinhaus. Sur la division pragmatique. Econometrica, 17:315-319, 1949. Supplement.

[Ste69] H. Steinhaus. Mathematical Snapshots. Oxford University Press, New York, 3rd edition, 1969.

[Str80] W. Stromquist. How to cut a cake fairly. The American Mathematical Monthly, 87(8):640-644, 1980 .

[Str07] W. Stromquist. Envy-free cake divisions cannot be found by finite protocols. In S. Brams, K. Pruhs, and G. Woeginger, editors, Dagstuhl Seminar 07261: "Fair Division”. Dagstuhl Seminar Proceedings, November 2007. Available on-line at http://drops.dagstuhl.de/opus/volltexte/2007/1220/pdf/07261.StromquistWalterPaper.1220.pdf.

[Tas03] A. Tasnádi. A new proportional procedure for the $n$-person cake-cutting problem. Economics Bulletin, 4(33):1-3, 2003.

[Var74] H. Varian. Equity, envy, and efficiency. Journal of Economic Theory, 9(1):63-91, 1974.

[Wel85] D. Weller. Fair division of a measurable space. Journal of Mathematical Economics, 14(1):5-17, 1985 .

[WS07] G. Woeginger and J. Sgall. On the complexity of cake cutting. Discrete Optimization, 4(2):213220, 2007. 